\title{
Effects of non-singular stresses on crack-tip fields for pressure-sensitive materials, Part 1: Plane strain case
}

\author{
M. KIM and J. PAN \\ Mechanical Engineering and Applied Mechanics, The University of Michigan, Ann Arbor, MI 48109, USA
}

Received 28 May 1993; accepted in revised form 6 May 1994

\begin{abstract}
Mode I near-tip stress fields for elastic perfectly plastic pressure-sensitive materials under plane strain and small-scale yielding conditions are presented. A Coulomb-type yield criterion described by a linear combination of the effective stress and the hydrostatic stress is adopted in the analysis. The finite element computational results sampled at the distance of a few crack opening displacements from the tip show that, as the pressure sensitivity increases, the magnitudes of the normalized radial and hoop stress ahead of the tip decrease, the total angular span of the singular plastic sectors decreases, and the angular span of the elastic sectors bordering the crack surfaces increases. When non-singular $T$ stresses are considered along the boundary layer of the small-scale yielding model, the near-tip stresses decrease as the $T$ stress decreases. The plastic zone shifts toward the crack surfaces as the $T$ stress increases. When the discontinuities of the radial stress and the out-of-plane normal stress along the border between the plastic sector and the elastic sector are allowed, the angular variations of the asymptotic crack-tip fields agree well with those of the finite element computations. Variation of the $Q$ stresses for pressure-sensitive materials can be found from the asymptotic solutions when the plastic zone size ahead of the tip is relatively larger than the crack opening displacement. In addition the $T$ stress is shown to have strong effects on the plastic zone sizes and shapes which could affect the toughening of pressure-sensitive materials.
\end{abstract}

\section{Introduction}

In the classical plasticity theory, it is assumed that hydrostatic pressure has no effect on material plastic deformation, and plastic dilatancy is neglected. But for many materials, such as soils, concrete, rocks and silicate glasses, macroscopic pressure-sensitive yielding and plastic volumetric deformation are exhibited. Toughened polymers, which are widely used in structures due to their light weight and relatively high strength and toughness, also show apparent pressure-sensitive yielding and plastic volumetric deformation, for example, see [1-3]. Pressure-sensitive yielding is also observed in zirconia-containing transformation toughened ceramics, for example, see [4-8]. It is considered that the pressure-sensitive yielding occurs from basic flow mechanism, cavitation and craze formation in some polymers and from phase transformation and microcracking in some ceramics.

Li and Pan [9, 10] made an attempt to study analytically pressure-sensitive yielding effects on asymptotic crack-tip fields based on a Coulomb-type yield criterion for powerlaw hardening materials and perfectly plastic materials under both plane strain and plane stress conditions. They found that the HRR-type asymptotic crack-tip fields [11-13] do exist for power-law hardening materials. They also found that the low-hardening solutions agree well with the corresponding perfectly plastic solutions. However, they could not find any HRR-type asymptotic solutions using the Runge-Kutta method for numerical integration and the shooting method for satisfying the boundary conditions when a pressure sensitivity factor $\mu$ reaches a limit value for each hardening exponent as the stress state ahead of the crack tip approaches the hydrostatic tension. Pan and Chen [14] used a finite element method for deformation plasticity power-law hardening materials to investigate the near-tip asymptotic 
fields under plane strain and small-scale yielding conditions. Their results showed that for $\mu$ larger than the limit value for each hardening exponent, the HRR field does exist and the stress state ahead of the tip stays in hydrostatic tension for a range of $\mu$. However, as $\mu$ becomes larger than a value near $\sqrt{3} / 2$, the HRR field breaks down and the near-tip field becomes oscillatory. Subsequently, Dong and Pan [15] investigated the crack-tip fields by finite element methods for elastic-plastic pressure-sensitive dilatant materials under plane strain and small-scale yielding conditions. Their results also indicated that the HRR-type asymptotic crack-tip fields exist beyond the limit value of the pressure sensitivity parameter for each hardening exponent for power-law hardening materials, and that elastic sectors of finite stresses exist and border the crack faces for elastic perfectly plastic materials, in contrast to the fully yielded solutions based on the slip-line theory proposed by $\mathrm{Li}$ and Pan [9]. Their perfectly plastic finite element solutions also indicated that when $\mu$ is larger than $\sqrt{3} / 2$, no asymptotic solutions can be found.

For elastic perfectly plastic Mises materials under plane strain conditions, Larsson and Carlsson [16] performed finite element computations for a variety of specimen geometries, such as center cracked panels and edge cracked bending specimens. They found that the computational results of the near-tip fields for the actual geometries showed larger discrepancies than those of the corresponding boundary layer formulation. In the boundary layer formulation, it is assumed that the $1 / \sqrt{r}$ singularity is dominant near a crack tip such as

$$
\sigma_{i j}=\frac{K}{\sqrt{2 \pi r}} f_{i j}(\theta)
$$

where $\sigma_{i j}$ represent the crack-tip stresses, $K$ is the mode I stress intensity factor, $r$ and $\theta$ are the polar coordinates centered at the crack tip, and $f_{i j}(\theta)$ are given by the linear elastic asymptotic solution. As suggested by Rice [17], Larsson and Carlsson [16] showed that the stress discrepancies were eliminated by introducing a non-singular stress term, which is the normal stress parallel to the crack line, along the boundary of the boundary layer formulation. The intensity of the non-singular stress term depends upon the geometry of the specimen and the type of loading. Accordingly, they modified the boundary layer formulation to compensate for the differences by including the non-singular stress ( $T$ stress) such as

$$
\sigma_{i j}=\frac{K}{\sqrt{2 \pi r}} f_{i j}(\theta)+T \delta_{i 1} \delta_{j 1}
$$

Rice [17] also gave very accurate estimations of plastic zone sizes and shapes based on a simple model where the effect of the $T$ stress can be included.

Bentegón and Hancock [18] studied the crack-tip fields for several crack geometries and for the boundary layer formulations with the small-strain assumption by finite element methods. Their results showed that the crack-tip stress fields under positive $T$ stresses were essentially similar to the HRR fields for hardening materials, but those under negative $T$ stresses deviated from the HRR fields. They also found similar results for non-hardening materials. Al-Ani and Hancock [19] showed a loss of $J$ dominance due to the geometry of the specimen from their large geometry change solutions. In their finite element computations, they used edge cracked bending and tension specimens with various ratios of crack length to width to obtain different $T$ stress values. They found that the $J$ dominance can be retained for the specimens where the $T$ stresses for small geometry change solutions are positive. Du and Hancock [20] employed the slip-line theory to interpret their finite element results and gave an empirical 
relation between the hydrostatic stress ahead of the crack tip as a function of the $T$ stress and the hydrostatic stress ahead of the crack tip without any $T$ stress. In Du and Hancock [20], the crack-tip stress fields are proposed to be represented by an incomplete Prandtl field with a constant stress sector, a centered fan sector and an elastic sector. They made no attempt to verify their proposed assembly of the asymptotic crack-tip structure for the Mises materials by their numerical solutions.

Here, we explore the combined effects of pressure-sensitive yielding and the non-singular $T$ stresses on crack-tip fields for elastic perfectly plastic pressure-sensitive materials under plane strain conditions and construct the corresponding asymptotic crack-tip stress fields. These asymptotic stress fields, due to the existence of an elastic sector, contain parameters which cannot be determined by the asymptotic analysis alone. We used the parameters from the results of finite element computations as the input for the asymptotic analysis. Then, the complete angular stress functions of the asymptotic analysis based on these input parameters are compared with those of the finite element solutions. Based on the asymptotic solutions and the computational results, we also explore the one parameter description of the $Q$ stress for pressure-sensitive materials in analogy to the $Q$ stress concept proposed by O'Dowd and Shih $[21,22]$. Finally, the implications and relevances of the results to the phase transformation toughening of ceramics are discussed.

\section{Governing equations}

A straight crack is considered as shown in Fig. 1 where both the Cartesian coordinates, $x_{1}$ and $x_{2}$, and the polar coordinates, $r$ and $\theta$, are centered at the crack tip. Rice [23] showed that due to the boundedness of the stresses as $r \rightarrow 0$, the asymptotic form of the equilibrium equations for perfectly plastic materials can be represented with respect to the polar coordinates as follows

$$
\begin{aligned}
& \sigma_{r r}-\sigma_{\theta \theta}+\frac{\mathrm{d} \sigma_{r \theta}}{\mathrm{d} \theta}=0, \\
& 2 \sigma_{r \theta}+\frac{\mathrm{d} \sigma_{\theta \theta}}{\mathrm{d} \theta}=0 .
\end{aligned}
$$

In order to incorporate the pressure-sensitive yielding, a simple Coulomb-type pressuresensitive yield criterion $[24,9,10]$, which is a linear combination of the effective stress $\sigma_{e}$ and the mean stress $\sigma_{m}$, is adopted here,

$$
\psi\left(\sigma_{i j}\right)=\sigma_{e}+\sqrt{3} \mu \sigma_{m}=\sigma_{g e}=\sigma_{o},
$$

where $\sigma_{e}=\left(3 s_{i j} s_{i j} / 2\right)^{1 / 2}, s_{i j}=\sigma_{i j}-\sigma_{m} \delta_{i j}$, and $\sigma_{m}=\sigma_{k k} / 3$. Here, $\delta_{i j}$ is the Kronecker delta and subscripts $i, j$ and $k$ have the range of 1 to 3 . In (5), $\psi\left(\sigma_{i j}\right)$ represents the yield surface in the stress space, and $\mu$ represents the pressure sensitivity of the material. Here $\mu$ is taken as a constant. In (5), $\sigma_{g e}$ is the generalized tensile effective stress. For perfectly plastic materials, $\sigma_{g e}$ is taken as a constant and is denoted as $\sigma_{o}$.

The components of the outward normal to the yield surface in the stress space are defined as

$$
P_{i j}=\frac{\partial \psi\left(\sigma_{i j}\right)}{\partial \sigma_{i j}}=\frac{3 s_{i j}}{2 \sigma_{e}}+\frac{\mu}{\sqrt{3}} \delta_{i j}
$$




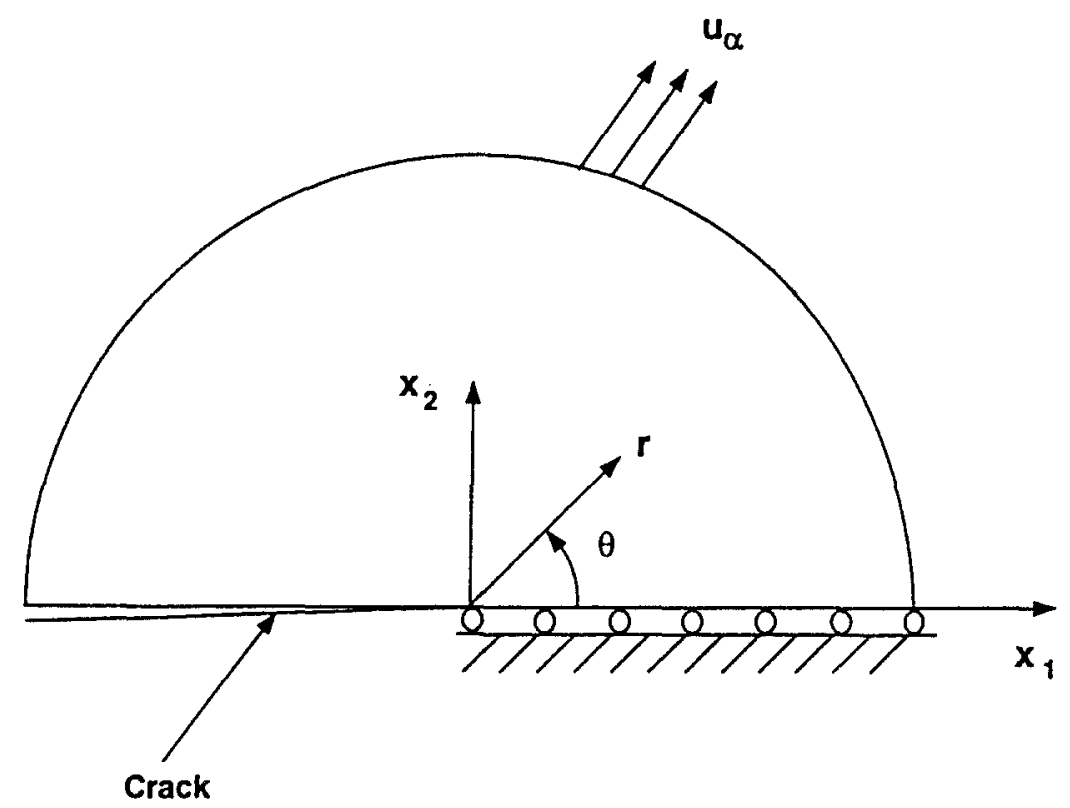

Fig. l. Coordinates coventions and model definition.

Within the context of the small-strain approach, the strain increments $d \epsilon_{i j}$ can be decomposed into an elastic part, $\mathrm{d} \epsilon_{i j}^{e}$, and a plastic part, $\mathrm{d} \epsilon_{i j}^{p}$. With Hooke's law of isotropic elasticity for the elastic strain increments and the assumption of the normality for the plastic strain increments, the strain increments can be written as

$$
\mathrm{d} \epsilon_{i j}=\mathrm{d} \epsilon_{i j}^{e}+\mathrm{d} \epsilon_{i j}^{p}=\frac{1+\nu}{E} \mathrm{~d} \sigma_{i j}-\frac{\nu}{E} \mathrm{~d} \sigma_{k k} \delta_{i j}+\mathrm{d} \lambda P_{i j}
$$

where $E$ is Young's modulus, $\nu$ is Poisson's ratio, and $\mathrm{d} \lambda$ is a proportionality factor. For transformation toughened ceramics, the experimental results suggested that the plastic strain increments follow the normality flow rule [7]. However, non-normality flow occurs in plastics, rocks and metals, see Drucker [1], Rudnicki and Rice [25] and Needleman and Rice [26]. For pressure-sensitive materials, a plastic potential was defined for non-normality flow in [27, 28]. Based on a perfectly plastic theory, the slip line fields around notch tips were constructed for pressure-sensitive materials and the effects of non-normality flow on slip lines were discussed in [27]. The effects of non-normality flow on the near-tip fields of blunted cracks in rubbertoughened epoxies were presented in [28]. Here we will concentrate on the crack-tip behavior for sharp cracks in pressure-sensitive materials with normality flow.

Assuming at least one in-plane component of the plastic strain increments is singular and $P_{\alpha \beta} \neq 0(\alpha, \beta=1,2)$ as $r \rightarrow 0$, then $\mathrm{d} \lambda \rightarrow \infty$ as $r \rightarrow 0$. Therefore, the plane strain condition $\mathrm{d} \epsilon_{33}=0$ requires

$$
P_{33}=\frac{3 s_{33}}{2 \sigma_{e}}+\frac{\mu}{\sqrt{3}}=0
$$

as $r \rightarrow 0$. Equation (8) also represents the plane strain condition for rigid perfectly plastic materials following the normality rule. Note that $\sigma_{e}=0$ represents a hydrostatic stress state 
and the stress state in yielding is at the vertex of the yield cone in the stress space. Then $P_{i j}$ cannot be uniquely defined.

From (8) the out-of-plane normal stress $\sigma_{33}$ can be expressed in terms of the three in-plane stresses with respect to the polar coordinates as

$$
\sigma_{33}=\frac{\sigma_{r r}+\sigma_{\theta \theta}}{2}-\frac{\mu}{\left[1-\mu^{2} / 3\right]^{1 / 2}}\left[\left(\frac{\sigma_{r r}-\sigma_{\theta \theta}}{2}\right)^{2}+\sigma_{r \theta}^{2}\right]^{1 / 2}
$$

Combining (5) and (9) gives

$$
\left[1-\frac{1}{3} \mu^{2}\right]^{1 / 2}\left[\left(\frac{\sigma_{r r}-\sigma_{\theta \theta}}{2}\right)^{2}+\sigma_{r \theta}^{2}\right]^{1 / 2}+\mu \frac{\sigma_{r r}+\sigma_{\theta \theta}}{2}=\frac{\sigma_{0}}{\sqrt{3}}
$$

From (9), we can see that $\sigma_{33}$ cannot be defined if $\mu=\sqrt{3}$. According to the general analysis of pressure-sensitive materials under plane strain conditions by Hill [29], the yield condition of (10) for $\mu \leqslant \sqrt{3} / 2$ can be transformed into a yield envelope in the Mohr plane. If two parameters, $\phi$ and $c$, are introduced as

$$
\sin \phi=\frac{\mu}{\left[1-\frac{1}{3} \mu^{2}\right]^{1 / 2}}
$$

and

$$
c=\frac{\sigma_{0}}{\sqrt{3}\left[1-\frac{4}{3} \mu^{2}\right]^{1 / 2}}
$$

then (10) can be expressed as

$$
\tau=c-\sigma \tan \phi
$$

Here, $\tau$ is the shear stress and $\sigma$ is the normal stress in the Mohr plane. Equation (13) is the well-known Coulomb criterion used in the slip-line theory in soil mechanics and concrete mechanics, for example, see [30]. Then, $\phi$ represents the angle of internal friction and $c$ represents the cohesion parameter.

\section{Singular plastic sector}

Rice [23] derived the governing equation for plastic sectors near the tip by combining the differential form of the yield condition and the asymptotic form of the equilibrium equations. The result which is applicable to the pressure-sensitive materials considered here is

$$
\frac{\mathrm{d}\left(\sigma_{11}+\sigma_{22}\right)}{\mathrm{d} \theta} P_{r r}+\frac{\mathrm{d} \sigma_{33}}{\mathrm{~d} \theta} P_{33}=0
$$

For singular plastic sectors with $P_{33}=0$ as $r \rightarrow 0$, (14) becomes

$$
\frac{\mathrm{d}\left(\sigma_{11}+\sigma_{22}\right)}{\mathrm{d} \theta} P_{r r}=0
$$


There exist two possible solutions to satisfy (15) [23]. One possible solution which represents a constant stress sector is

$$
\frac{\mathrm{d}\left(\sigma_{11}+\sigma_{22}\right)}{\mathrm{d} \theta}=0
$$

or,

$$
\begin{aligned}
& \sigma_{11}=\text { constant } \\
& \sigma_{22}=\text { constant } \\
& \sigma_{12}=\text { constant } \\
& \sigma_{33}=\text { constant }
\end{aligned}
$$

All the stresses with respect to the Cartesian coordinates are constants in this sector. The constants are to be determined by the yield condition, the plane strain condition expressed in (8), and the boundary conditions.

The other possible solution which represents a centered fan sector is

$$
P_{r r}=\frac{3 s_{r r}}{2 \sigma_{e}}+\frac{\mu}{\sqrt{3}}=0
$$

According to [29], $P_{r r}=0$ indicates that the radial lines represent a family of characteristic lines of the hyperbolic stress equations when $\mu<\sqrt{3} / 2$. The other family of the stress characteristics are in general not orthogonal to the radial lines unless $\mu=0$.

\subsection{CONSTANT STRESS SECTOR}

The shear stress referring to the Cartesian coordinates in the constant stress sector ahead of the crack tip as shown in Fig. 2 should be zero to satisfy the symmetry condition of the mode I crack-tip fields. Then,

$$
\sigma_{11}=\text { constant }, \quad \sigma_{22}=\text { constant }, \quad \sigma_{12}=0 .
$$

When we express (10) with respect to the Cartesian coordinates, with $\sigma_{12}=0$ we can easily express $\sigma_{22}$ in terms of $\sigma_{11}$ as

$$
\sigma_{22}=\frac{2 \sigma_{0}}{\sqrt{3}\left(\left[1-\mu^{2} / 3\right]^{1 / 2}+\mu\right)}+\frac{\left[1-\mu^{2} / 3\right]^{1 / 2}-\mu}{\left[1-\mu^{2} / 3\right]^{1 / 2}+\mu} \sigma_{11} .
$$

The out-of-plane stress $\sigma_{33}$ can then be obtained when (9) is expressed with respect to the Cartesian coordinates. There is another constant stress sector in the assembly of the crack-tip sectors as shown in Fig. 2(b). The assembly of the crack-tip sectors will be detailed later in this paper. The stresses in this sector must be determined by the boundary conditions, the yield condition and the plane strain condition. 


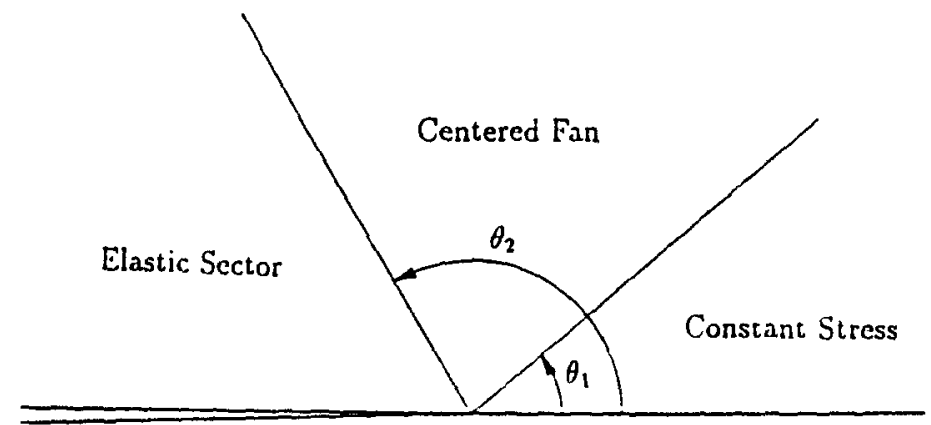

(a)

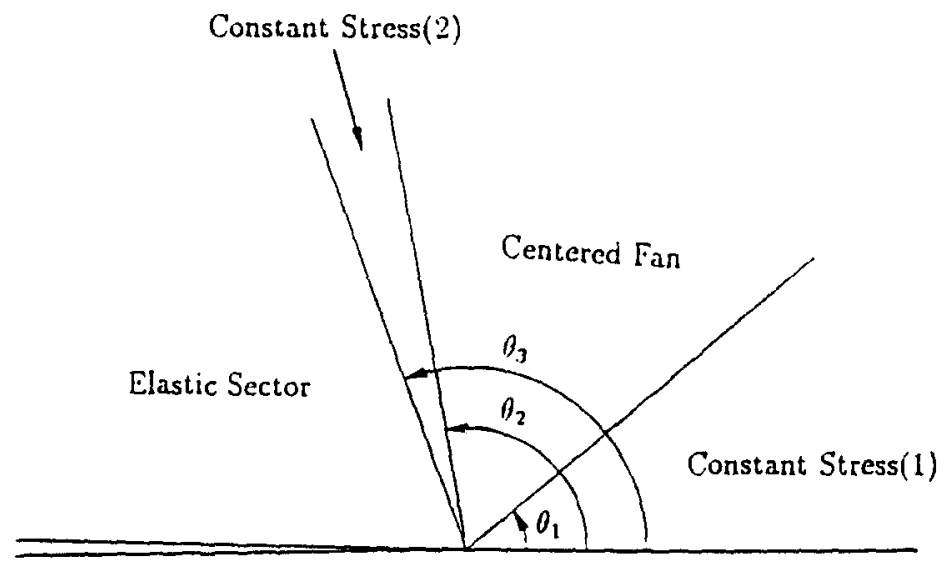

(b)

Fig. 2. The assemblies of the asymptotic crack-tip fields. (a) Two singular plastic sectors and an elastic sector facing the crack surface, (b) Three singular plastic sectors and an elastic sector facing the crack surface.

\subsection{CENTERED FAN SECTOR}

Since the conditions of $P_{r r}=0$ and $P_{33}=0$ give $\sigma_{r r}=\sigma_{33}$ in the centered fan sector, (21) can be rewritten as

$$
\sigma_{r r}-\sigma_{\theta \theta}+2 \mu\left[\frac{\left(\sigma_{r r}-\sigma_{\theta \theta}\right)^{2}}{3}+\sigma_{r \theta}^{2}\right]^{1 / 2}=0 .
$$

If we differentiate the above equation with respect to $\theta$, we have

$$
\sigma_{r r}^{\prime}-\sigma_{\theta \theta}^{\prime}+\mu\left[\frac{\left(\sigma_{r r}-\sigma_{\theta \theta}\right)^{2}}{3}+\sigma_{r \theta}^{2}\right]^{-1 / 2}\left(\frac{2}{3}\left(\sigma_{r r}-\sigma_{\theta \theta}\right)\left(\sigma_{r r}^{\prime}-\sigma_{\theta \theta}^{\prime}\right)+2 \sigma_{r \theta} \sigma_{r \theta}^{\prime}\right)=0,
$$

where $\sigma_{i j}^{\prime}=\mathrm{d} \sigma_{i j} / \mathrm{d} \theta$. From (3), (4) and (25) the governing equations for the stress fields in this sector can be rewritten as

$$
\begin{aligned}
& \sigma_{\theta \theta}^{\prime}=-2 \sigma_{r \theta}, \\
& \sigma_{r \theta}^{\prime}=\sigma_{\theta \theta}-\sigma_{r r},
\end{aligned}
$$




$$
\begin{aligned}
& \sigma_{r r}^{\prime}=\frac{\left(1+2 \mu Z\left(\sigma_{r r}-\sigma_{\theta \theta}\right) / 3\right) \sigma_{\theta \theta}^{\prime}-2 \mu Z \sigma_{r \theta} \sigma_{r \theta}^{\prime}}{1+2 \mu Z\left(\sigma_{r r}-\sigma_{\theta \theta}\right) / 3}, \\
& \sigma_{33}=\sigma_{r r},
\end{aligned}
$$

where $Z=\left[\left(\sigma_{r r}-\sigma_{\theta \theta}\right)^{2} / 3+\sigma_{r \theta}^{2}\right]^{-1 / 2}$. Then we can use the Runge-Kutta numerical integration scheme to integrate (26)-(28) in order to find the stress fields in the centered fan sector. As $\mu \rightarrow 0,(24)$ is reduced to $\sigma_{r r}=\sigma_{\theta \theta}$ which represents one characteristic equation of the centered fan sector for the Mises materials under plane strain conditions. The numerical results, using (26)-(29), showed that as $\mu \rightarrow 0$, the stress state in the centered fan sector converged to that for the Mises materials.

In the following, we derive the analytical solutions for centered fan sectors. The values of $\mu$ are about $0.02 \sim 0.06$ for steels [31], $0.1 \sim 0.25$ for polymers [32], and $0.55 \sim 0.77$ for transformation toughened ceramics $[6,7]$. However, for $\mathrm{CeO}_{2}$-TZP ceramics, $\mu$ can be as high as 0.93 [8]. Here, we restrict our study to $\mu<\sqrt{3} / 2$ since asymptotic crack-tip fields can be found under this condition [15].

First, the relation of the three in-plane stress components can be derived from (8) and (21) for $\mu<\sqrt{3} / 2$ as

$$
\sigma_{r r}-\sigma_{\theta \theta}=\mp \frac{2 \mu}{\left[1-\frac{4}{3} \mu^{2}\right]^{1 / 2}} \sigma_{r \theta}
$$

where we take ' - ' when $\sigma_{r \theta}>0$ and ' + ' when $\sigma_{r \theta}<0$. From (3) and (30), we have

$$
\frac{\mathrm{d} \sigma_{r \theta}}{\mathrm{d} \theta} \mp \frac{2 \mu}{\left[1-\frac{4}{3} \mu^{2}\right]^{1 / 2}} \sigma_{r \theta}=0
$$

When we integrate (31), we set the lower integration limit at $\theta_{0}$ and we denote $\sigma_{r \theta}=\sigma_{r \theta}^{0}$ at $\theta=\theta_{0}$. Then

$$
\sigma_{r \theta}=\sigma_{r \theta}^{0} \exp \left( \pm \frac{2 \mu\left(\theta-\theta_{0}\right)}{\left[1-\frac{4}{3} \mu^{2}\right]^{1 / 2}}\right) .
$$

Here, $\sigma_{r \theta}^{0}$ and $\theta_{0}$ can be found to satisfy the boundary conditions. Then $\sigma_{\theta \theta}$ can be calculated from (4) for $\mu \neq 0$ as

$$
\sigma_{\theta \theta}=-\frac{\left[1-\frac{4}{3} \mu^{2}\right]^{1 / 2}}{\mu} \sigma_{r \theta}^{0} \exp \left( \pm \frac{2 \mu\left(\theta-\theta_{0}\right)}{\left[1-\frac{4}{3} \mu^{2}\right]^{1 / 2}}\right)+C
$$

where $C$ is an integration constant which can be determined to satisfy the yield condition. Equations (30), (32) and (33) can be used to find $\sigma_{r r}$ as

$$
\sigma_{r r}=\left(-\frac{\left[1-\frac{4}{3} \mu^{2}\right]^{1 / 2}}{\mu}-\frac{2 \mu}{\left[1-\frac{4}{3} \mu^{2}\right]^{1 / 2}}\right) \sigma_{r \theta}^{0} \exp \left( \pm \frac{2 \mu\left(\theta-\theta_{0}\right)}{\left[1-\frac{4}{3} \mu^{2}\right]^{1 / 2}}\right)+C
$$

If $\mu \neq 0$, the integration constant $C$ can be determined as $C=\sigma_{0} / \sqrt{3} \mu$ for the stress states described in (32), (33) and (34) to satisfy the yield condition in (5). 
As $\mu \rightarrow 0,(32,(33)$ and (34) cannot be used to determine the stress states in centered fan sectors since in the present form $\sigma_{\theta \theta}$ and $\sigma_{r r}$ will not converge to finite values. In order to determine the stress states as $\mu \rightarrow 0$, we expand the exponential term near $\mu=0$ as

$$
\exp \left( \pm \frac{2 \mu\left(\theta-\theta_{0}\right)}{\left[1-\frac{4}{3} \mu^{2}\right]^{1 / 2}}\right)=1 \pm 2 \mu\left(\theta-\theta_{0}\right)+O\left[\mu^{2}\right]
$$

If we substitute this first order expansion in $\mu$ into (32), (33) and (34) for $\mu \rightarrow 0$,

$$
\begin{aligned}
& \sigma_{r \theta}=\sigma_{r \theta}^{0}, \\
& \sigma_{\theta \theta}=\mp 2 \sigma_{r \theta}^{0}\left(\theta-\theta_{0}\right)-\frac{\sigma_{r \theta}^{0}}{\mu}+\frac{\sigma_{0}}{\sqrt{3} \mu}, \\
& \sigma_{r r}=\mp 2 \sigma_{r \theta}^{0}\left(\theta-\theta_{0}\right)-\frac{\sigma_{r \theta}^{0}}{\mu}+\frac{\sigma_{0}}{\sqrt{3} \mu}, \\
& \sigma_{33}=\sigma_{r r} .
\end{aligned}
$$

As $\mu \rightarrow 0$, the stress states will exist only if $\sigma_{r \theta}^{0}=\sigma_{0} / \sqrt{3}$. Therefore, the stress states of (36) through (39) converge to those of the centered fan sector for the Mises materials.

\section{Elastic sector}

The finite element results $[34,35]$ showed that finite stress elastic sectors exist near the crack surfaces at physically reasonable radial distances to the tip for elastic perfectly plastic materials. The stresses in the elastic sectors are obtained to satisfy the compatibility equation and the equilibrium equations [35] as

$$
\begin{aligned}
& \sigma_{r r}=2 A_{8}+2 A_{9} \theta-2 A_{10} \cos 2 \theta-2 A_{11} \sin 2 \theta, \\
& \sigma_{\theta \theta}=2 A_{8}+2 A_{9} \theta+2 A_{10} \cos 2 \theta+2 A_{11} \sin 2 \theta, \\
& \sigma_{r \theta}=-A_{9}+2 A_{10} \sin 2 \theta-2 A_{11} \cos 2 \theta, \\
& \sigma_{33}=\nu\left(\sigma_{r r}+\sigma_{\theta \theta}\right) .
\end{aligned}
$$

Here, $A_{8}, A_{9}, A_{10}$, and $A_{11}$ are constants which can be determined by the boundary conditions. The generalized effective tensile stress $\sigma_{g e}$ is now expressed as

$$
\sigma_{g e}=\left[\frac{1}{2}\left(\left(\sigma_{r r}-\sigma_{\theta \theta}\right)^{2}+\left(\sigma_{\theta \theta}-\sigma_{33}\right)^{2}+\left(\sigma_{33}-\sigma_{r r}\right)^{2}\right)+3 \sigma_{r \theta}^{2}\right]^{1 / 2}+\sqrt{3} \mu \sigma_{m} .
$$

The generalized tensile effective stress $\sigma_{g e}$ in (44) has a different form from that in (10) since in singular plastic sectors $\sigma_{33}$ is determined by $P_{33}=0$ whereas in elastic sectors $\sigma_{33}=\nu\left(\sigma_{r r}+\sigma_{\theta \theta}\right)$. 


\section{Boundary conditions}

The traction along the border between any two sectors must be continuous. This gives

$$
\sigma_{\theta \theta}\left(\theta_{i}^{-}\right)=\sigma_{\theta \theta}\left(\theta_{i}^{+}\right)
$$

and

$$
\sigma_{r \theta}\left(\theta_{i}^{-}\right)=\sigma_{r \theta}\left(\theta_{i}^{+}\right)
$$

where $\theta_{i}^{-}$and $\theta_{i}^{+}$, measured in the counterclockwise sense, represent the angles just before and after the border angle $\theta_{i}$, respectively. The traction free conditions on the crack surfaces are

$$
\sigma_{\theta \theta}( \pm \pi)=\sigma_{r \theta}( \pm \pi)=0
$$

\section{Assembly of stationary crack-tip fields}

For perfectly plastic materials, the solutions of the stationary asymptotic crack-tip fields are not unique. Under mode I and plane strain conditions, the fully yielded Prandtl field has been proposed and verified for the Mises materials $[36,37]$. The fully yielded crack-tip field for pressure-sensitive materials based on the Coulomb yield criterion has been proposed in [9]. However, the finite element solutions of [15] indicate that there exist elastic sectors bordering the crack surfaces at physically reasonable distances to the tip for $0<\mu<\sqrt{3} / 2$. We therefore construct the asymptotic crack-tip fields which consist of a constant stress sector, a centered fan sector and an elastic sector for pressure-sensitive materials. Because of the symmetry of the mode I crack-tip fields, only a constant stress sector can be placed ahead of the crack tip, and is followed by a centered fan sector. Figure 2(a) shows the structure of this assembly. In this figure $\theta_{1}$ and $\theta_{2}$ represent the angles delineating these sectors.

Due to the equilibrium equations, $\sigma_{\theta \theta}$ and $\sigma_{r \theta}$ must be continuous along the border between the constant stress sector and the centered fan sector. The other two stress components, $\sigma_{r r}$ and $\sigma_{33}$, in the constant stress sector can be obtained to satisfy the plane strain condition for singular plastic sectors in (8) and the yield condition in (5). In the centered fan sector, an extra condition $P_{r r}=0$ must be satisfied. In the Appendix, we will show that when $\sigma_{\theta \theta}$ and $\sigma_{r \theta}$ are continuous along the border between a constant stress sector and a centered fan sector, $\sigma_{r r}$ and $\sigma_{33}$ must be continuous.

At $\theta_{1}$ the stress state in the constant stress sector should satisfy (21) because of the above argument. When $\sigma_{r \theta}>0, \theta_{1}$ can be determined uniquely for a given pressure sensitivity $\mu$ as

$$
\theta_{1}=\frac{1}{2} \tan ^{-1}\left(\frac{\left[1-\frac{4}{3} \mu^{2}\right]^{1 / 2}}{\mu}\right)
$$

The angle $\theta_{1}$ found from (48) gives the same results as those in Li and Pan [9] in which they applied the slip line theory of the soil mechanics to rigid perfectly plastic materials. We can also see that as $\mu$ approaches $0, \theta_{1}$ approaches $45^{\circ}$ which agrees with the solution for the Mises materials.

We now extend the full stress continuity condition to the border of the plastic sector and the elastic sector based on the observations of the finite element solutions. From the structure 
shown in Fig. 2(a) we can find two possible solutions for a given pressure sensitivity. For example, for $\mu=0.4$ one solution gave $\theta_{1}=32.86^{\circ}, \theta_{2}=41.58^{\circ}, \sigma_{r r}(0)=0.1953 \sigma_{0}$ and $\sigma_{\theta \theta}(0)=0.9225 \sigma_{0}$. The other solution gave $\theta_{1}=32.86^{\circ}$ and $\theta_{2}=121.82^{\circ}, \sigma_{r r}(0)=$ $1.0905 \sigma_{0}$ and $\sigma_{\theta \theta}(0)=1.2961 \sigma_{0}$. The angular stress distributions of the latter solution are close to those of the finite element results shown in Fig. 5(a). However, we must recognize that the angular stress distribution at the given radius to the tip must be a function of the $T$ stress for a given pressure sensitivity. We cannot accept the two solutions as the only two possibilities under different $T$ stresses.

From a close examination of the finite element solutions, we notice a possible plastic sector between the centered fan sector and the elastic sector. For simplicity, this sector is now considered to have the stress field of a constant stress sector. Therefore, the structure of the crack-tip fields, starting from the front of the crack, is in the order of a constant stress sector, a centered fan sector, another constant stress sector and finally an elastic sector facing the crack surface as shown in Fig. 2(b). In this figure, $\theta_{1}, \theta_{2}$ and $\theta_{3}$ represent the angles delineating these sectors.

If one more constant stress sector is included as shown in Fig. 2(b), compared to Fig. 2(a) we have to determine four more stress components $\sigma_{11}, \sigma_{22}, \sigma_{33}$, and $\sigma_{12}$ in this sector and one more angle. We can only have four equations from the full stress continuity condition along the border between the second constant stress sector and the centered fan sector. Thus, if we use this assembly and the full stress continuity condition between the second constant stress sector and the elastic sector, we are short of one condition. If we input the value of $\sigma_{11}$ from the finite element computations, the results showed that the total angular span of the singular plastic sectors becomes much larger than that of the finite element solutions. Therefore, we decided to impose only the continuity of $\sigma_{\theta \theta}$ and $\sigma_{r \theta}$, and allow the discontinuity of $\sigma_{r r}$ and $\sigma_{33}$ along the border between the second constant stress sector and the elastic sector. Then, we are short of three conditions. Therefore, we chose the values of $\theta_{2}, \theta_{3}$ (as defined in Fig. 2(b)) and $\sigma_{11}$ in front of the crack tip from the finite element solutions and used them as the input parameters to determine the crack-tip stress fields.

As described in Section 3.1, once $\sigma_{11}$ is given, the stress state in the first constant stress sector can be determined. At $\theta_{1}$ which can be obtained from (48), the stress state of the first constant stress sector can then be used to start the integration of (26), (27) and (28). A sixth-order Runge-Kutta numerical integration scheme was used to find the stress state of the centered fan sector from $\theta_{1}$ to $\theta_{2}$. Here, $\theta_{2}$ was chosen from the finite element solution. At $\theta_{2}$, the stress state in the second constant stress sector can be determined by imposing the continuity of $\sigma_{\theta \theta}$ and $\sigma_{r \theta}$. As shown in the Appendix, when $\sigma_{\theta \theta}$ and $\sigma_{r \theta}$ are continuous between the two singular plastic sectors, $\sigma_{r r}$ and $\sigma_{33}$ must also be continuous. The stress state at $\theta_{3}$ can be obtained from that of the second constant stress sector. For the elastic sector, the traction free conditions in (47) can be used to express $A_{8}$ and $A_{9}$ in terms of $A_{10}$ and $A_{11}$ in (41) and (42). Then $A_{8}$ and $A_{9}$ can be determined from the continuity of $\sigma_{\theta \theta}$ and $\sigma_{r \theta}$ at $\theta_{3}$. When the analytical solutions in (32), (33) and (34) for the centered fan sector were used, the numerical results were the same as those from the Runge-Kutta numerical integration scheme.

\section{Finite element computational model}

A crack in a circular domain under mode I loading is considered. Due to the symmetry, the upper half of the circular domain is considered as shown in Fig. 1. Twenty equally spaced elements are placed in the angular direction and twenty-four semi-circular rings of elements 
are placed in the radial direction. Crack-tip elements with collapsed nodes are employed to simulate $1 / r$ singularity of the strains. The nodal points in the radial direction are generated by a logarithmic scale, and the size of the crack-tip elements in the radial direction is set at one ten-thousandth of the outer radius. In the models, there are 500 eight-node elements and 1591 nodal points.

The displacement fields in terms of $K$ and $T$ near a crack in linear elastic materials were given as the boundary conditions along the circumference of the semi-circular domain as

$$
u_{\alpha}=\frac{K}{2 G} \frac{\sqrt{r}}{2 \pi} f_{\alpha}(\theta, \nu)+\frac{T r}{E} g_{\alpha}(\theta, \nu) .
$$

Here, $G$ represents the shear modulus, and $f_{\alpha}(\theta, \nu)$ and $g_{\alpha}(\theta, \nu)$ are the dimensionless functions given by the linear elastic solutions. Here, $\alpha$ has the range of 1 and 2 . In order to maintain the proportional loading conditions throughout the loading, the biaxiality parameter (Leevers and Radon [38]) was adopted as

$$
B=\frac{T \sqrt{\pi} a}{K}
$$

where $a$ is the crack length. Now, the loading can be applied through mode I stress intensity factor $K$. The nodal points along the crack ligament were constrained in the vertical direction to simulate the symmetry of the mode I crack-tip fields. In the computations, $E / \sigma_{0}=500$ and $\nu=0.3$ were used.

The finite element program used in the computations was developed in [15]. A displacementbased finite element method with an iterative procedure based on a modified Newton-Raphson method was used in the analysis. Within the context of the small-strain approach, an incremental plasticity theory with the pressure-sensitive yield criterion and the normality flow rule was incorporated to govern the plastic deformation.

Based on the small-strain assumption the crack tip is assumed to remain sharp during the deformation in the asymptotic analysis. However, when finite deformation is considered, crack tip blunting must be taken into account. Therefore, in our small-strain finite element analysis, the sampling radius for the stresses and strains should be appropriately far from the crack tip, where the effect of the crack-tip blunting almost vanishes. Inside of the region bordered by this radius, the deformation is so large that the small-strain approach is no longer applicable. McMeeking [39] performed a finite element study based on the finite deformation approach under plane strain and small-scale yielding conditions. He showed that for the distance from the tip, which is greater than two or three times of the crack-tip opening displacement, the deviation of the stress fields from those of the small-strain theory becomes unnoticeable. For power-law hardening materials, Shih [40] gave the relation between the crack-tip opening displacement and the $J$-integral under small-scale yielding conditions as

$$
\delta_{t}=\mathrm{d}_{n} \frac{J}{\sigma_{0}}
$$

where $\delta_{t}$ is a crack-tip opening displacement, $J$ represents the $J$-integral [36], $\sigma_{0}$ is a reference stress, and $d_{n}$ is a constant as a function of the hardening exponent. For perfectly plastic materials Shih [40] suggested $d_{n}=1$, although he inferred from the observations of several cracked geometries under fully yielded conditions that there is no unique relationship between 
$J$ and the crack-tip opening displacement for non-hardening materials [41]. For linear elastic materials the relation between $J$ and $K$ is

$$
J=\frac{K^{2}}{E^{\prime}},
$$

where $E^{\prime}=E /\left(1-\nu^{2}\right)$ under plane strain conditions. Now, we can relate the remote applied $K$ and the crack-tip opening displacement. This will lead us to determine the sampling radius at a certain load level. As McMeeking [39], Shih [40], and O'Dowd and Shih [21, 22] suggested, we took the sampling radius at $r=2 J / \sigma_{0}$, where $J$ is calculated from (52). From the biaxiality factor, we can decide the $B$ value corresponding to the sampling radius. The crack-tip stress fields for three different values of $\mu, 0,0.4$, and 0.8 , under three different values of $T / \sigma_{0}, 0.5$, 0 , and -0.5 , were studied.

\section{Computational results and asymptotic solutions}

Figures 3, 5 and 7 show the angular distributions of the normalized stresses under different $T$ stresses for $\mu=0,0.4$, and 0.8 , respectively. In these figures, the symbols represent the finite element solutions at the integration points located at $r=2 J / \sigma_{0}$ and the solid lines represent the corresponding asymptotic solutions. Schematic plots of the assemblies of the various crack-tip sectors under different $T$ stresses are shown in Figs. 4, 6 and 8 for $\mu=0,0.4$, and 0.8 , respectively. In these figures, 'C.S.' represents constant stress sector, 'C.F.' represents centered fan sector and 'Elastic' denotes elastic sector.

In Fig. 3(a) for $\mu=0$ and $T / \sigma_{0}=0.5$, the value of $\sigma_{\theta \theta} / \sigma_{0}$ ahead of the crack tip is 2.94 which is about 1 percent lower than 2.97 of the Prandtl solution [36]. The stress field appears to be fully yielded as shown in Fig. 3(a) and agrees very well with the asymptotic solution. In Fig. 3(b) for $\mu=0$ and $T / \sigma_{0}=0$, the value of $\sigma_{\theta \theta} / \sigma_{0}$ ahead of the crack tip is 2.81 which is lower than that of the Prandtl solution. For this case, the crack-tip field is not fully yielded. Figure 3(c) shows the angular distributions of the normalized stresses for $\mu=0$ and $T / \sigma_{0}=-0.5$. For this case, the value of $\sigma_{\theta \theta} / \sigma_{0}$ ahead of the crack tip is 2.20 which is about 26 percent lower than that of the Prandtl solution. The out-of-plane stress component $\sigma_{33}$ in the constant stress sector ahead of the tip shows some discrepancies from the corresponding asymptotic solution. This was also observed by Tracey [42]. The reason for this phenomenon is that the radius of the plastic zone ahead of the crack tip is not significantly larger than $2 J / \sigma_{0}$ for our pressure-insensitive Mises materials with the ratio of $\sigma_{0} / E=1 / 500$. If we go closer to the crack tip, we do see in Fig. 15 that $\sigma_{33}$ of the finite element solution approaches to that of the asymptotic analysis. When the plastic zone size ahead of the crack tip becomes significantly larger than $2 J / \sigma_{0}$, the discrepancies decrease. In Fig. 4 for $\mu=0$, we can see that as $T / \sigma_{0}$ decreases, the angular span of the centered fan sector decreases and the angular span of the elastic sector increases. This trend was also observed in [20]. The angular span of the constant stress sector ahead of the crack tip is not changed by the $T$ stress as indicated in (48).

Figures 5(a), 5(b) and 5(c) show the angular distributions of the normalized stresses for $\mu=0.4$ under $T / \sigma_{0}=0.5,0$ and -0.5 , respectively. It should be noted that the value of $\sigma_{\theta \theta} / \sigma_{0}$ ahead of the crack tip is 1.29 for $T / \sigma_{0}=0.5,1.26$ for $T / \sigma_{0}=0$, and 1.09 for $T / \sigma_{0}=-0.5$. It should also be noted that the radial stress near the crack surface for $T / \sigma_{0}=-0.5$ is compressive as shown in Fig. 5(c) for $T / \sigma_{0}=-0.5$. In Fig. 6(a) for $\mu=0.4$ and $T / \sigma_{0}=0.5$, the angular span of the second constant stress sector is estimated 

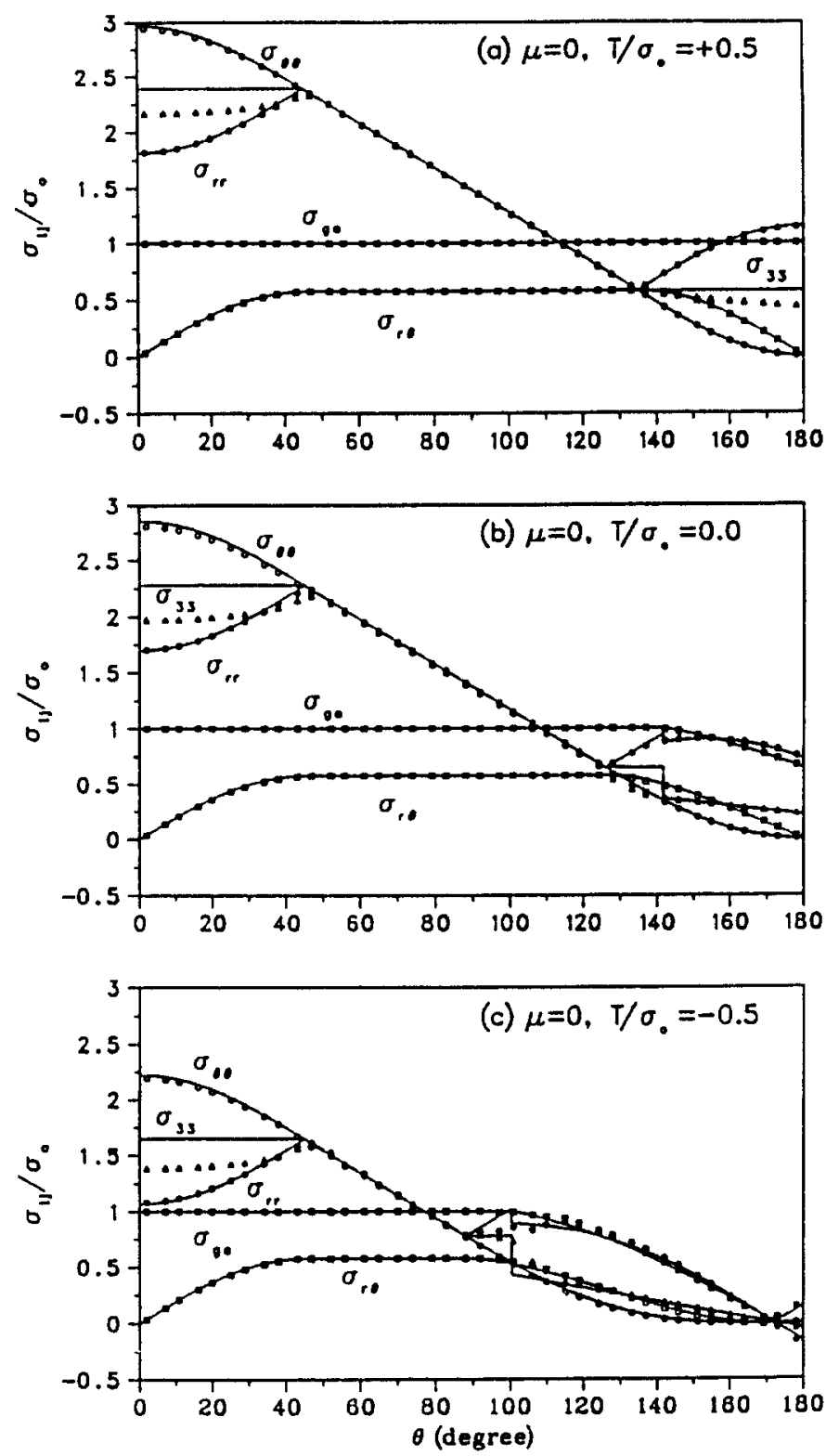

Fig. 3. The angular variations of the crack-tip stresses normalized by $\sigma_{0}$ at $2 J / \sigma_{0}$ for $\mu=0$. (a) $T / \sigma_{0}=0.5$, (b) $T / \sigma_{0}=0$, (c) $T / \sigma_{0}=-0.5$. The symbols represent the finite element solutions and the solid lines represent the asymptotic solutions based on the assembly shown in Fig. 2(b).

as small as $2.8^{\circ}$ which is significantly less than those of Figs. $6(\mathrm{~b})$ and (c) for $T / \sigma_{0}=0$ and -0.5 , respectively. Since the angular span of the second constant stress sector is so small for $T / \sigma_{0}=0.5$, the stress field of the finite element solution therefore is close to one of the two fully determined solutions (see Fig. 2(a)) as discussed earlier.

Figures $7(\mathrm{a})$, (b) and (c) show the angular distributions of the normalized stresses for $\mu=0.8$ under $T / \sigma_{0}=0.5,0$, and -0.5 , respectively. The values of $\sigma_{\theta \theta} / \sigma_{0}$ ahead of the crack tip are 0.69 for $T / \sigma_{0}=0.5,0.72$ for $T / \sigma_{0}=0$, and 0.71 for $T / \sigma_{0}=-0.5$. The variation of the values of $\sigma_{\theta \theta} / \sigma_{0}$ ahead of the crack tip due to the $T$ stress is small for 

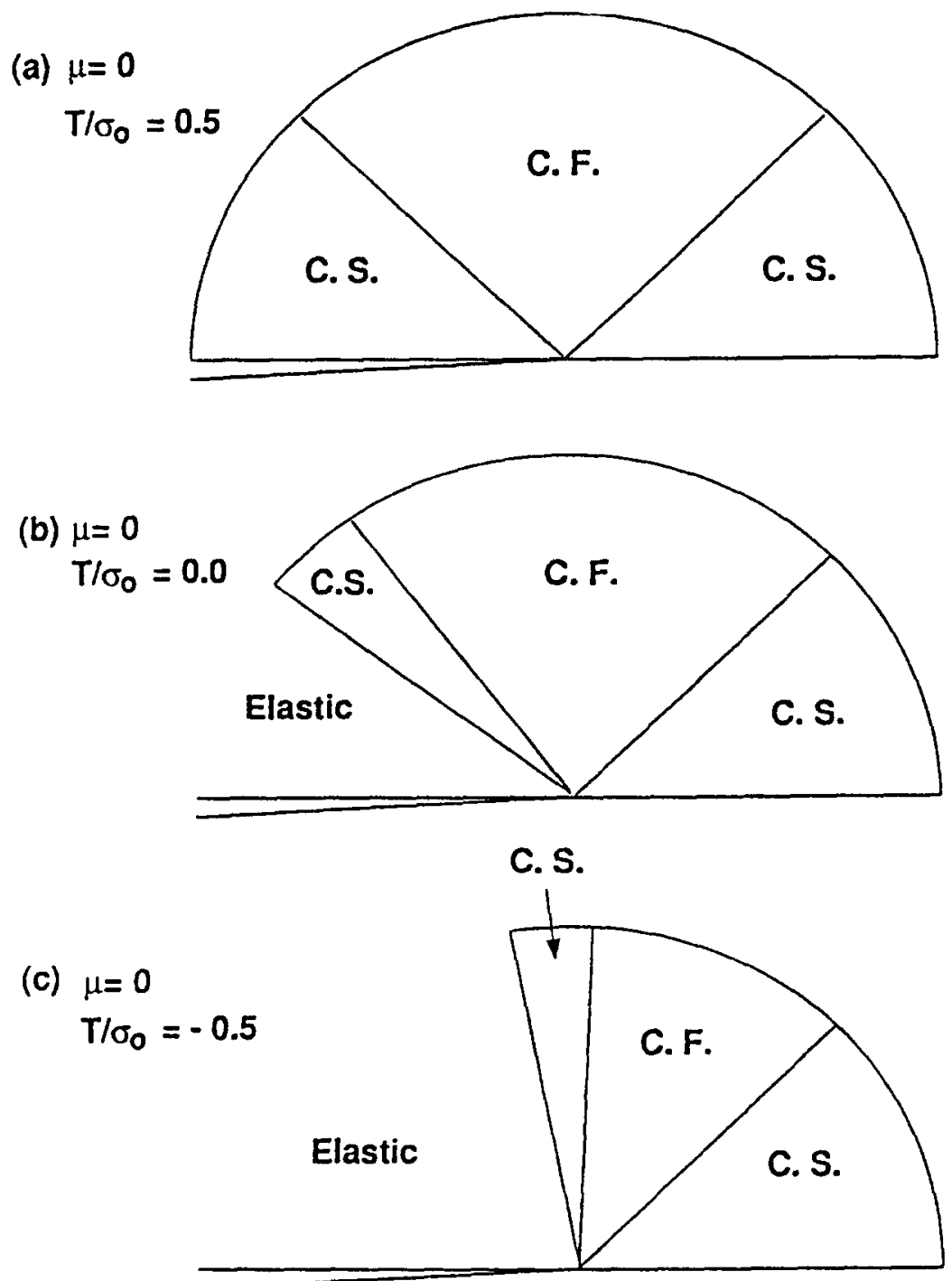

Fig. 4. The schematic plots of the assembly of various sectors for $\mu=0$. (a) $T / \sigma_{0}=0.5$, (b) $T / \sigma_{0}=0$, (c) $T / \sigma_{0}=-0.5$.

$\mu=0.8$. From our finite element results the value of $\sigma_{\theta \theta}$ is slightly less than that of $\sigma_{r r}$ for $T / \sigma_{0}=0.5$. This can be seen more clearly in Fig. 17(a). From the asymptotic solutions for $T / \sigma_{0}=0.5$ the stress state ahead of the crack tip is close to pure hydrostatic stress state and $\sigma_{\theta \theta}$ is still slightly larger than that of $\sigma_{r r}$. The result that the value of $\sigma_{\theta \theta}$ is slightly less than that of $\sigma_{r r}$ may come from the accumulation of errors during the computations. Schematic plots of the assemblies of the various crack-tip sectors under different $T$ stresses are shown in Fig. 8 for $\mu=0.8$. In this figure, we can see that the angular span of the elastic stress sector is larger than those of $\mu=0$ and 0.4 under the same value of $T / \sigma_{0}$. The angular spans of the second constant stress sectors for $T / \sigma_{0}=0.5,0$, and -0.5 are $35.9^{\circ}, 13.1^{\circ}$, and $39^{\circ}$, respectively.

Figures 9(a), (b) and (c) show the normalized plastic zone sizes and shapes from the finite element computations for $\mu=0$. The normalized plastic zone radius ahead of the 

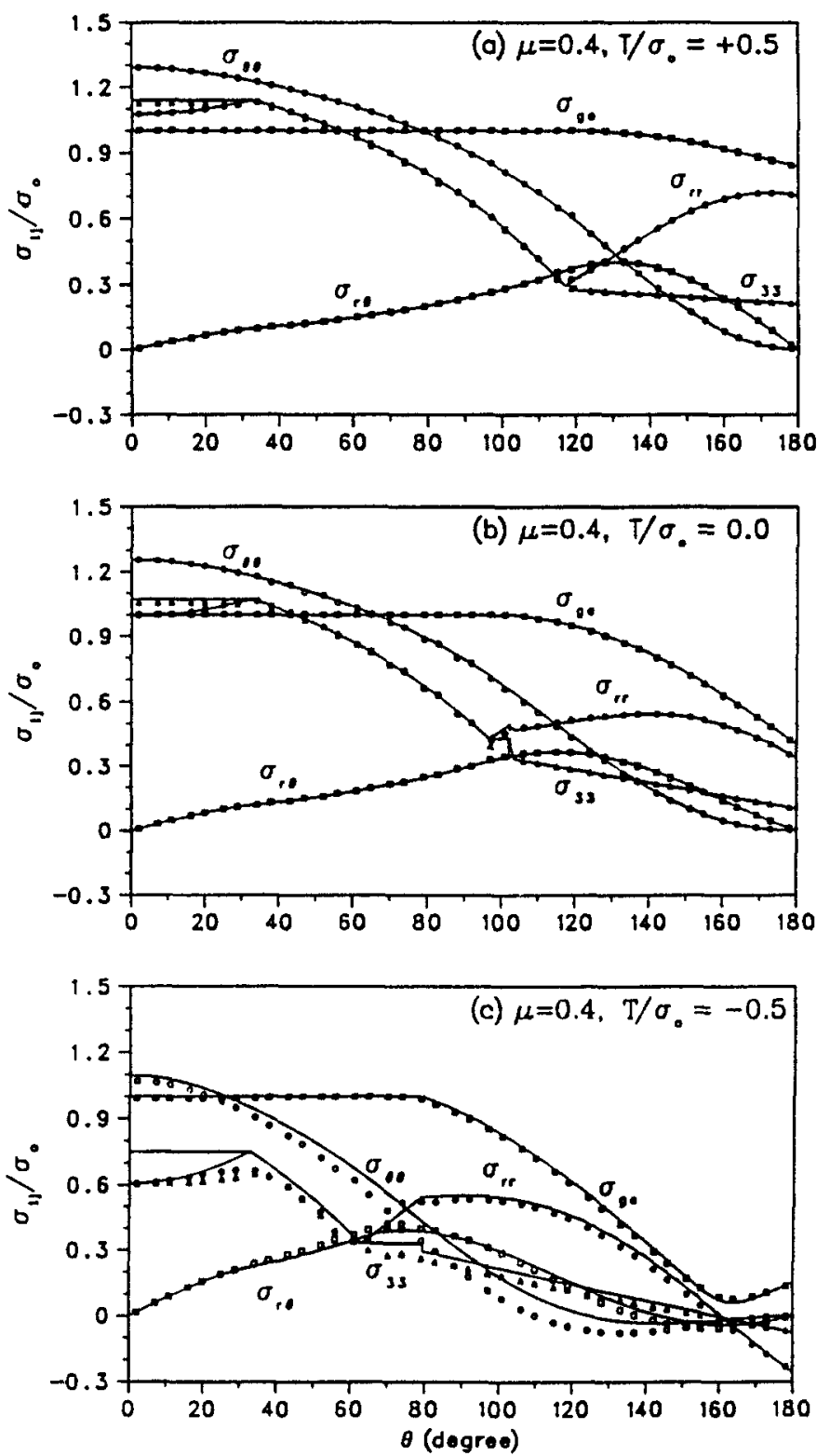

Fig. 5. The angular variations of the crack-tip stresses normalized by $\sigma_{0}$ at $2 J / \sigma_{0}$ for $\mu=0.4$. (a) $T / \sigma_{0}=0.5$, (b) $T / \sigma_{0}=0$, (c) $T / \sigma_{0}=-0.5$. The symbols represent the finite element solutions and the solid lines represent the asymptotic solutions based on the assembly shown in Fig. 2(b).

tip, $r /\left(K / \sigma_{0}\right)^{2}$, for $T / \sigma_{0}=0.5$ is 0.034 which is almost the same as that for $T / \sigma_{0}=0$. For $T / \sigma_{0}=-0.5$, the normalized plastic zone radius ahead of the crack tip is 0.027 . The normalized plastic zone shapes are completely different for different values of $T / \sigma_{0}$. The maximum plastic zone radius for $T / \sigma_{0}=-0.5$ is about three times those for $T / \sigma_{0}=0$ and 0.5 . The maximum normalized plastic zone radius is located at $97^{\circ} \sim 109^{\circ}$ under $T / \sigma_{0}=0.5$, $55^{\circ} \sim 82^{\circ}$ under $T / \sigma_{0}=0$, and $56^{\circ}$ under $T / \sigma_{0}=-0.5$. These results are consistent with those of [16], [18] and [21.22]. 
(a)

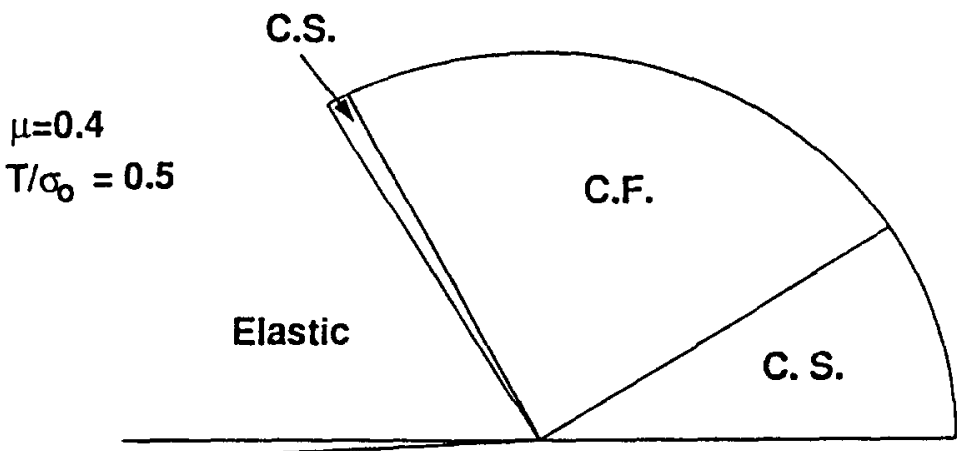

(b) $\mu=0.4$

$T / \sigma_{0}=0.0$

C.S.

(c) $\mu=0.4$

$T / \sigma_{0}=-0.5$

Elastic

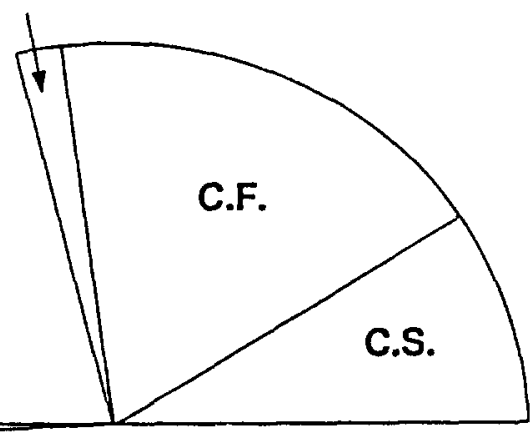

Elastic

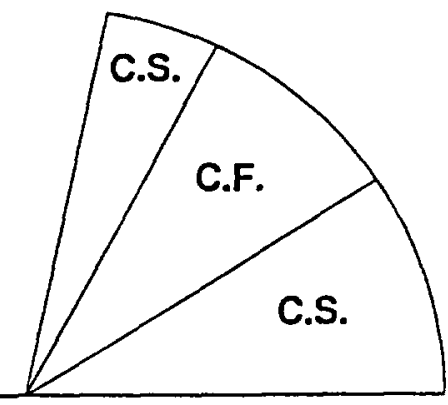

Fig. 6. The schematic plots of the assembly of various sectors for $\mu=0.4$. (a) $T / \sigma_{0}=0.5$, (b) $T / \sigma_{0}=0$, (c) $T / \sigma_{0}=-0.5$.

The normalized plastic zone sizes and shapes are shown in Fig. 10 for $\mu=0.4$ under $T / \sigma_{0}=0.5,0$, and -0.5 . The normalized plastic zone radii ahead of the crack tip are 0.299 , 0.252 , and 0.113 under $T / \sigma_{0}=0.5,0$, and -0.5 , respectively. As $T$ varies, the trend of the shape change of the plastic zones is the same as that of the $\mu=0$ case. As shown in Fig. 10 (a) for $T / \sigma_{0}=0.5$, the maximum normalized plastic zone radius is 0.446 and it is located at $88^{\circ} \sim 101^{\circ}$. Among these outmost integration points of the plastic zone shown in Fig. 10(a) the highest plastic strain is observed at $97^{\circ}$. As shown in Fig. 10(b) for $T / \sigma_{0}=0$, the maximum normalized plastic zone radius is 0.299 and it is located at $25^{\circ} \sim 52^{\circ}$. Among these outmost integration points the largest plastic strain is observed at $43^{\circ}$. As shown in Fig. 10 (c) for $T / \sigma_{0}=-0.5$, the maximum normalized plastic zone radius increases to 0.561 and 

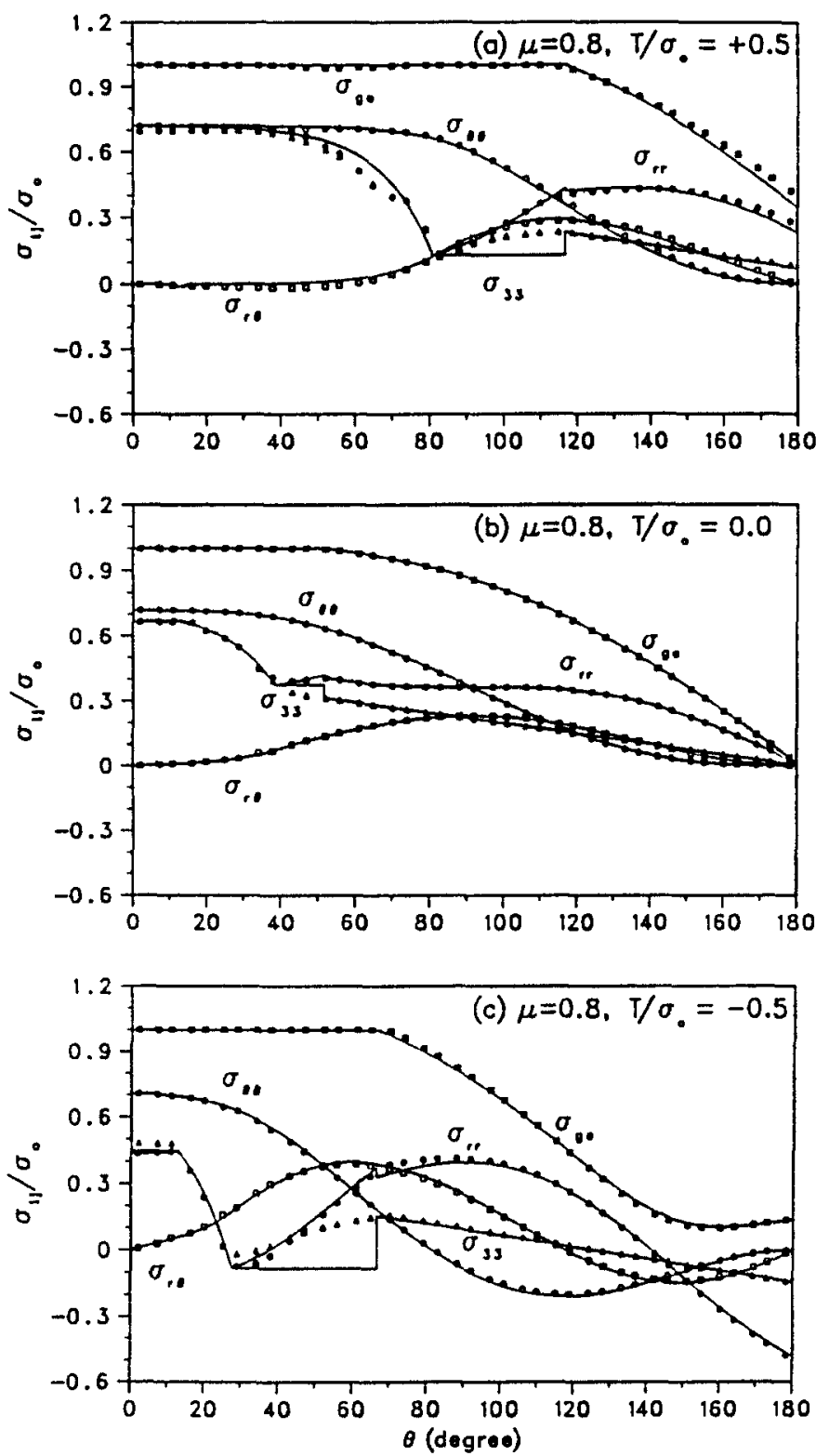

Fig. 7. The angular variations of the crack-tip stresses normalized by $\sigma_{0}$ at $2 J / \sigma_{0}$ for $\mu=0.8$. (a) $T / \sigma_{0}=0.5$, (b) $T / \sigma_{0}=0$, (c) $T / \sigma_{0}=-0.5$. The symbols represent the finite element solutions and the solid lines represent the asymptotic solutions based on the assembly shown in Fig. 2(b).

it is located at $29^{\circ} \sim 43^{\circ}$. Among these outmost integration points the largest plastic strain is observed at $34^{\circ}$.

Figures 11(a), (b) and (c) show the normalized plastic zone sizes and shapes for $\mu=0.8$. In Fig. 11(a) for $T / \sigma_{0}=0.5$, the maximum normalized plastic zone radius is observed at $79^{\circ} \sim 82^{\circ}$ and $1.8^{\circ} \sim 54^{\circ}$. In Figs. 11(b) and (c) for $T / \sigma_{0}=0$ and -0.5 , the maximum normalized plastic zone radii are located ahead of the crack tip for both cases. The maximum normalized plastic zone radius is located at $1.8^{\circ} \sim 16.1^{\circ}$ for $T / \sigma_{0}=0$ and $1.8^{\circ} \sim 25.1^{\circ}$ for 
(a)

$$
\begin{aligned}
& \mu=0.8 \\
& T / \sigma_{0}=0.5
\end{aligned}
$$

Elastic

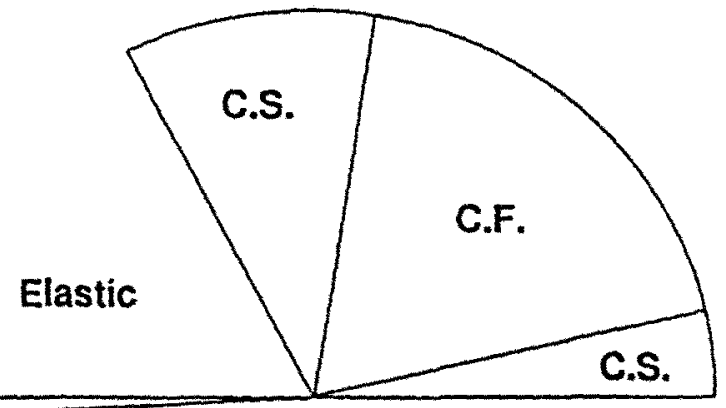

(b) $\mu=0.8$

$$
T / \sigma_{0}=0.0
$$

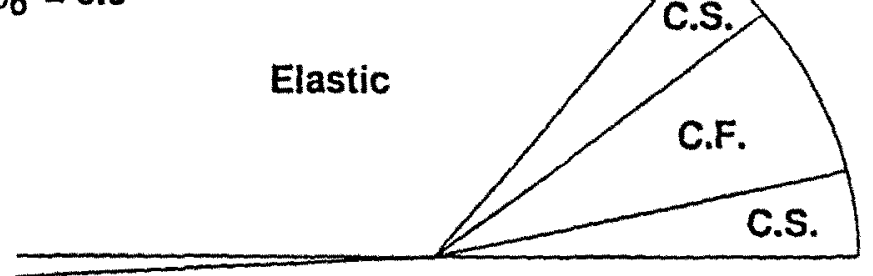

(c) $\mu=0.8$

$$
T / \sigma_{0}=-0.5
$$

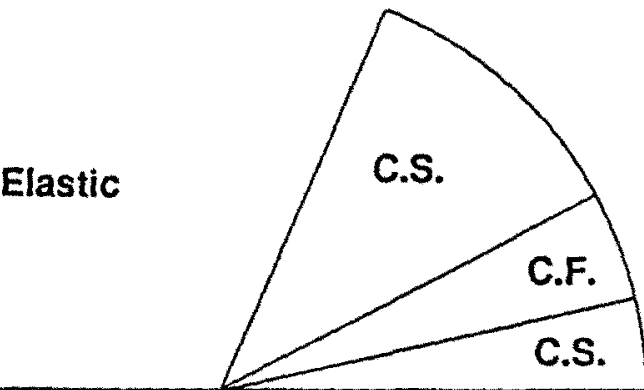

Fig. 8. The schematic plots of the assembly of various sectors for $\mu=0.8$. (a) $T / \sigma_{0}=0.5$, (b) $T / \sigma_{0}=0$, (c) $T / \sigma_{0}=-0.5$.

$T / \sigma_{0}=-0.5$. The maximum normalized plastic zone radii are $1.868,0.666$, and 0.666 for $T / \sigma_{0}=0.5,0$, and -0.5 , respectively.

In summary, the effects of the $T$ stress on the plastic zone sizes and shapes for pressuresensitive materials are rather complex. However, there are general trends, the plastic zone tends to move toward the crack surface as the $T$ stress increases for the same $\mu$ value. On the other hand, as $\mu$ increases, the normalized plastic zone shifts to the front of the crack tip.

Figures 12,13 and 14 show the normalized plastic zone sizes and shapes obtained from the linear asymptotic stress fields characterized by $K$ and $T$ under plane strain conditions for $\mu=0,0.4$ and 0.8 , respectively. Although full-field finite element computations should be used to determine the plastic zone sizes and shapes, the plastic zones shown in Figs. 12,13 and 14 can give us some references for comparisons with the plastic zones from the finite element 

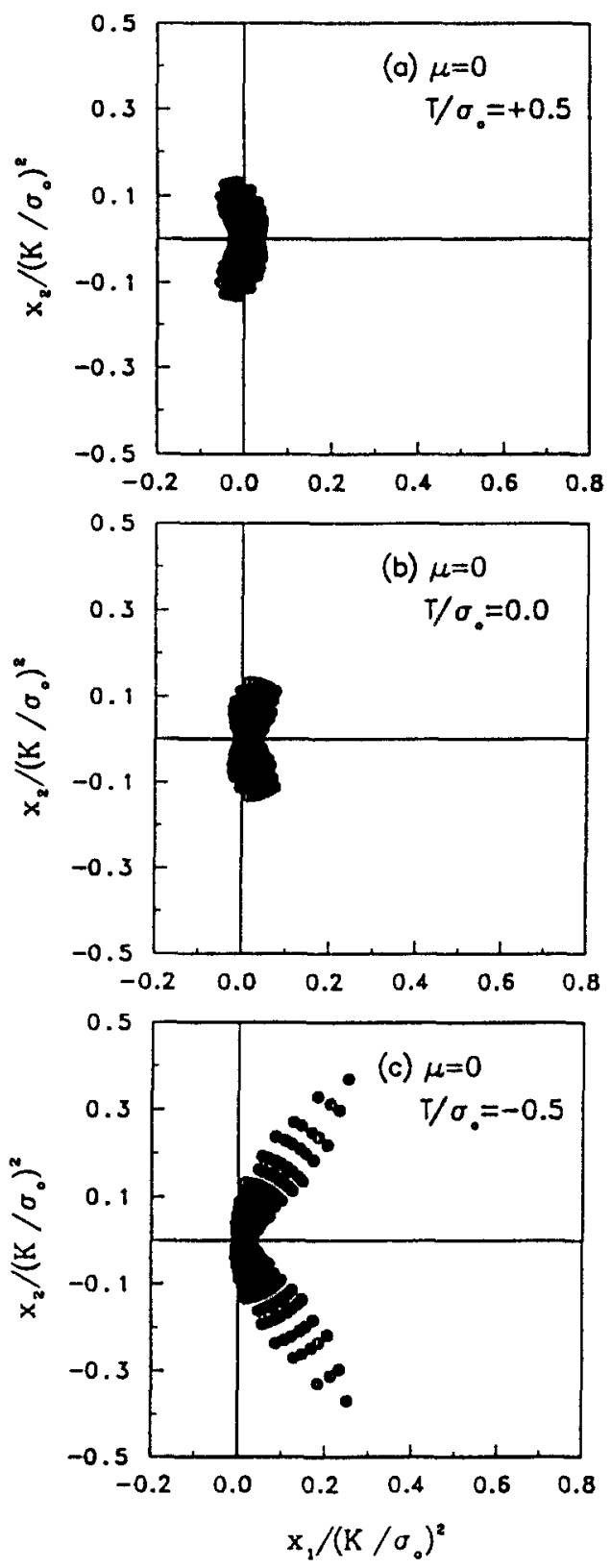

Fig. 9. The normalized plastic zone sizes and shapes from the finite element computations for $\mu=0$. (a) $T / \sigma_{0}=0.5$, (b) $T / \sigma_{0}=0$, (c) $T / \sigma_{0}=-0.5$.

computations. Figures 12(a), (b) and (c) show the normalized plastic zones, for the Mises materials under $T / \sigma_{0}=0.5,0$ and -0.5 , respectively. In general, the trends of the shapes and sizes of the normalized plastic zones in Fig. 12 agree with those in Fig. 9. When both the pressure sensitivity and the $T$ stress are considered, the shapes and sizes of the normalized plastic zones obtained from the linear elastic asymptotic stress fields deviate from those of the finite element computations. If we compare the shapes and sizes of the normalized plastic zones for $\mu=0.4$ in Fig. 13 with those in Fig. 10, we can clearly see the differences. For $\mu=0.8$ in Fig. 14(a) under $T / \sigma_{0}=0.5$, the size of the normalized plastic zone ahead of the 

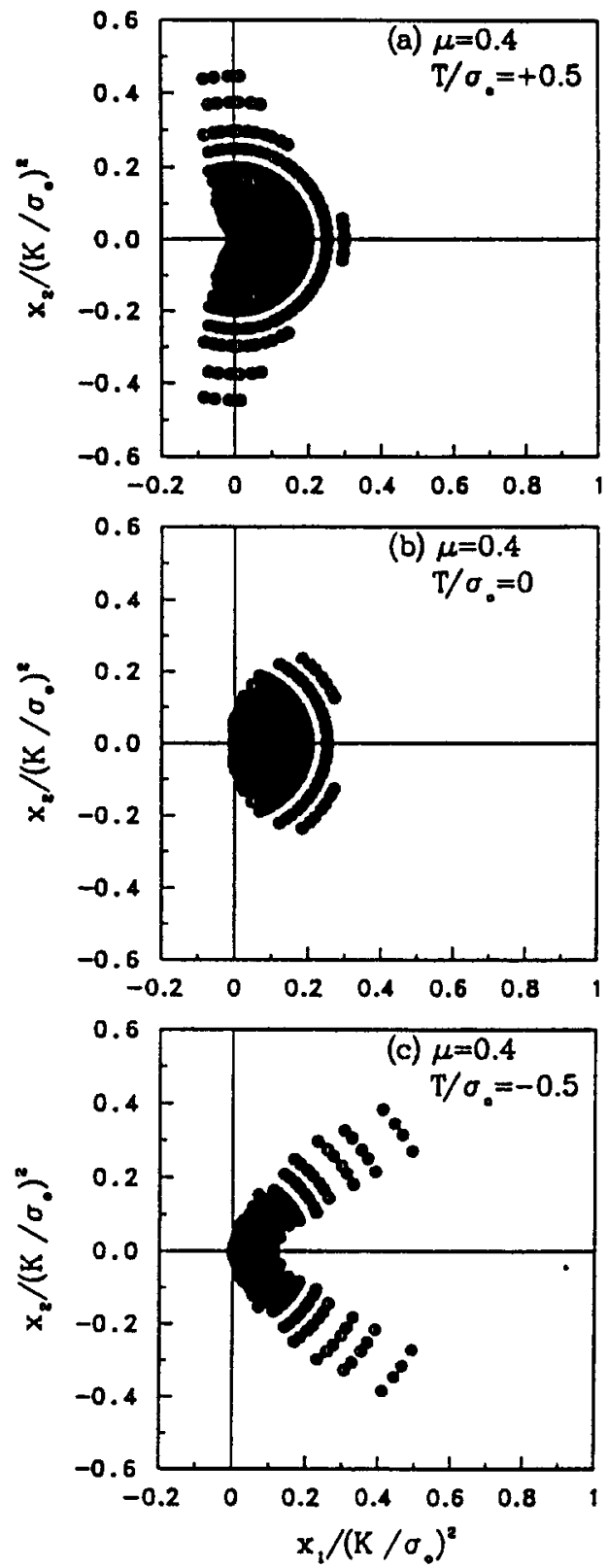

Fig. 10. The normalized plastic zone sizes and shapes from the finite element computations for $\mu=0.4$. (a) $T / \sigma_{0}=0.5$, (b) $T / \sigma_{0}=0$, (c) $T / \sigma_{0}=-0.5$.

crack tip is twice as large as that of the finite element computation shown in Fig. 11(a). In Figs. 14(b) and (c) for $\mu=0.8$, the shapes of the normalized plastic zones are quite different from those of the finite element computations shown in Figs. 11(b) and (c). However, the normalized plastic zones from the elastic asymptotic stress fields and those from the finite element computations showed the same general trends of the effects of the $T$ stress and the pressure sensitivity.

The normalized stresses $\sigma_{i j} / \sigma_{0}$ ahead of the crack tip at $\theta=1.9^{\circ}$ as functions of the normalized radius $r /\left(J / \sigma_{0}\right)$ are shown in Figs. 15, 16 and 17 for $\mu=0,0.4$ and 0.8 , 


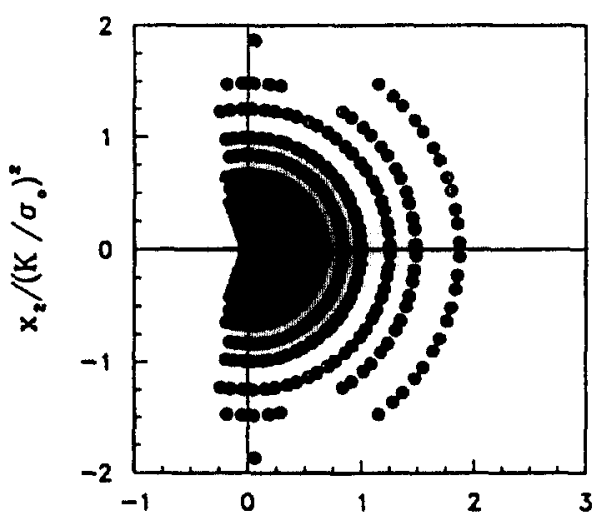

(a) $\mu=0.8$

$T / \sigma_{.}=+0.5$

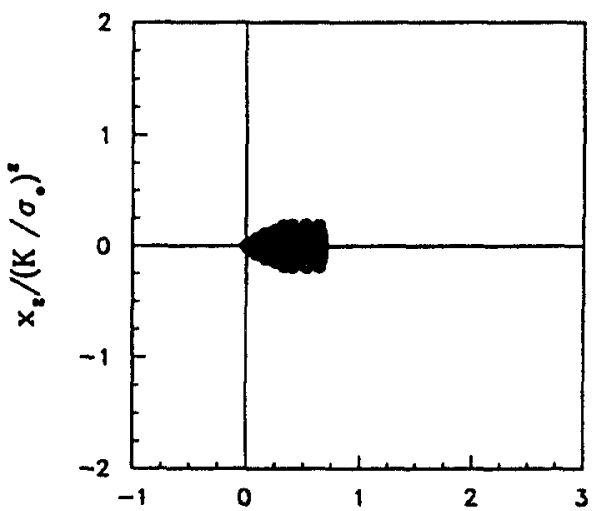

(b) $\mu=0.8$

$T / \sigma_{0}=0$

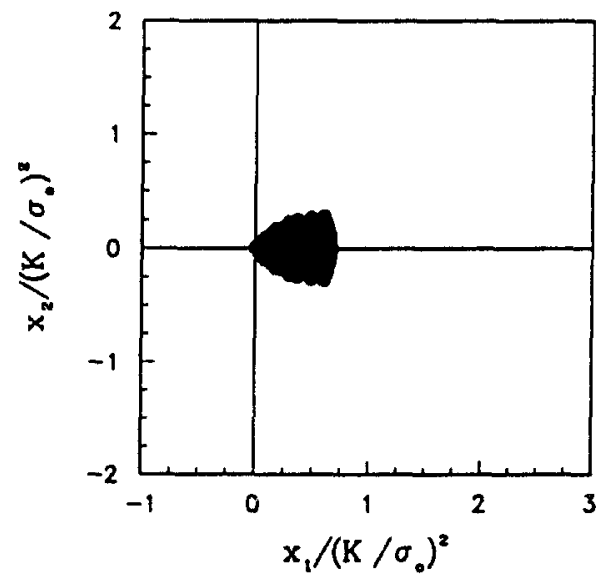

(c) $\mu=0.8$

$T / \sigma_{0}=-0.5$

Fig. 11. The normalized plastic zone sizes and shapes from the finite element computations for $\mu=0.8$. (a) $T / \sigma_{0}=0.5$, (b) $T / \sigma_{0}=0$, (c) $T / \sigma_{0}=-0.5$.

respectively. As shown in Fig. 15 for $\mu=0, \sigma_{11}$ and $\sigma_{22}$ level off whereas $\sigma_{33}$ still increases as $r$ decreases. The plastic zone size ahead of the tip is not large when compared with $2 J / \sigma_{0}$. Therefore the normalized $\sigma_{33}$ shown in Fig. 3, taken at $2 J / \sigma_{0}$, does not agree well with the corresponding asymptotic solution. In Figs. 16 and 17 for $\mu=0.4$ and 0.8 , the plastic zone sizes ahead of the tip are large when compared with $2 J / \sigma_{0}$. Therefore the normalized stresses generally level off at $2 J / \sigma_{0}$ except in the case of Fig. 16(c) for $\mu=0.4$ and $T / \sigma_{0}=-0.5$. This observation has important implications to the generalization of the $Q$ stress for pressuresensitive materials. 

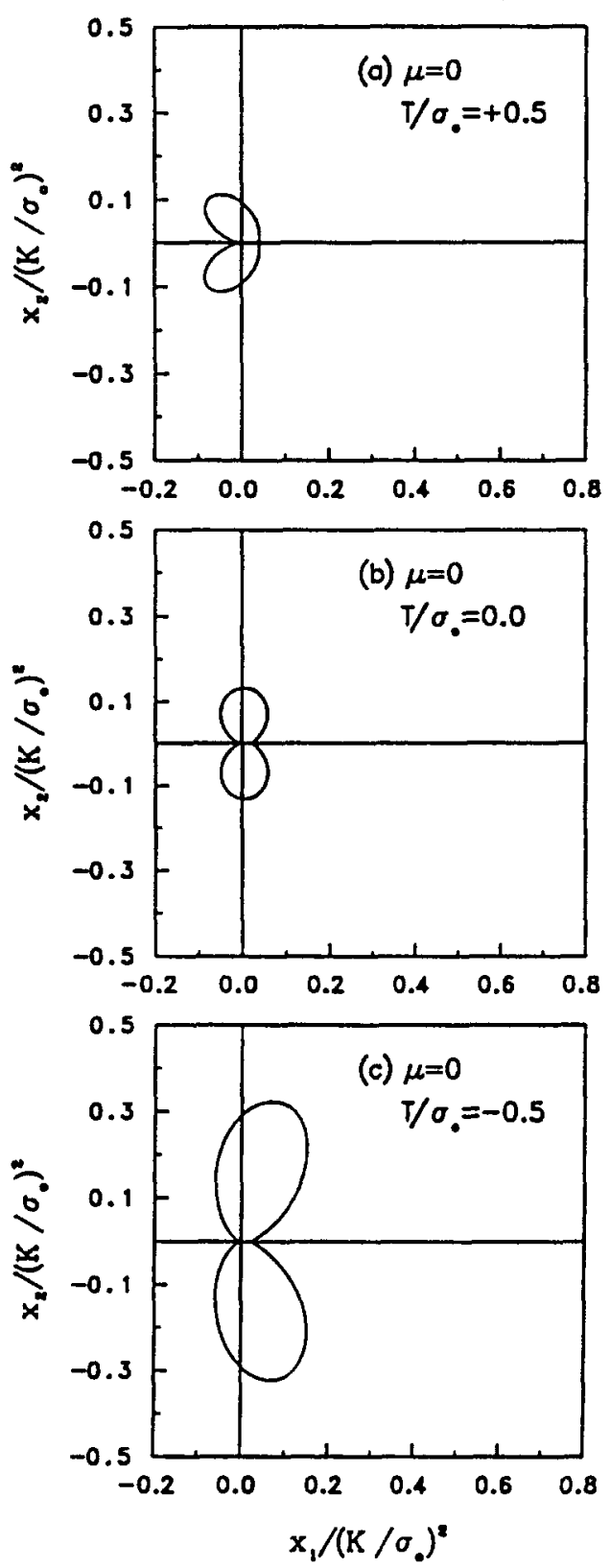

Fig. 12. The normalized plastic zone sizes and shapes obtained from the linear elastic asymptotic fields for $\mu=0$. (a) $T / \sigma_{0}=0.5$, (b) $T / \sigma_{0}=0$, (c) $T / \sigma_{0}=-0.5$.

\section{Discussions and conclusions}

As presented in O'Dowd and Shih [21,22], $J$ sets the size scale of the crack-tip process zone over which large stresses and strains develop, while $Q$ scales the near-tip stress distribution relative to a high triaxiality reference stress state for pressure-insensitive incompressible Mises materials. They proposed two possible reference fields to determine the $Q$ stress. One is the field under small-scale yielding conditions and $T=0$. The other is the asymptotic HRR fields for power-law hardening materials. As plastic hardening decreases to zero, the HRR field for power-law hardening materials approaches the fully yielded Prandtl field. Therefore 

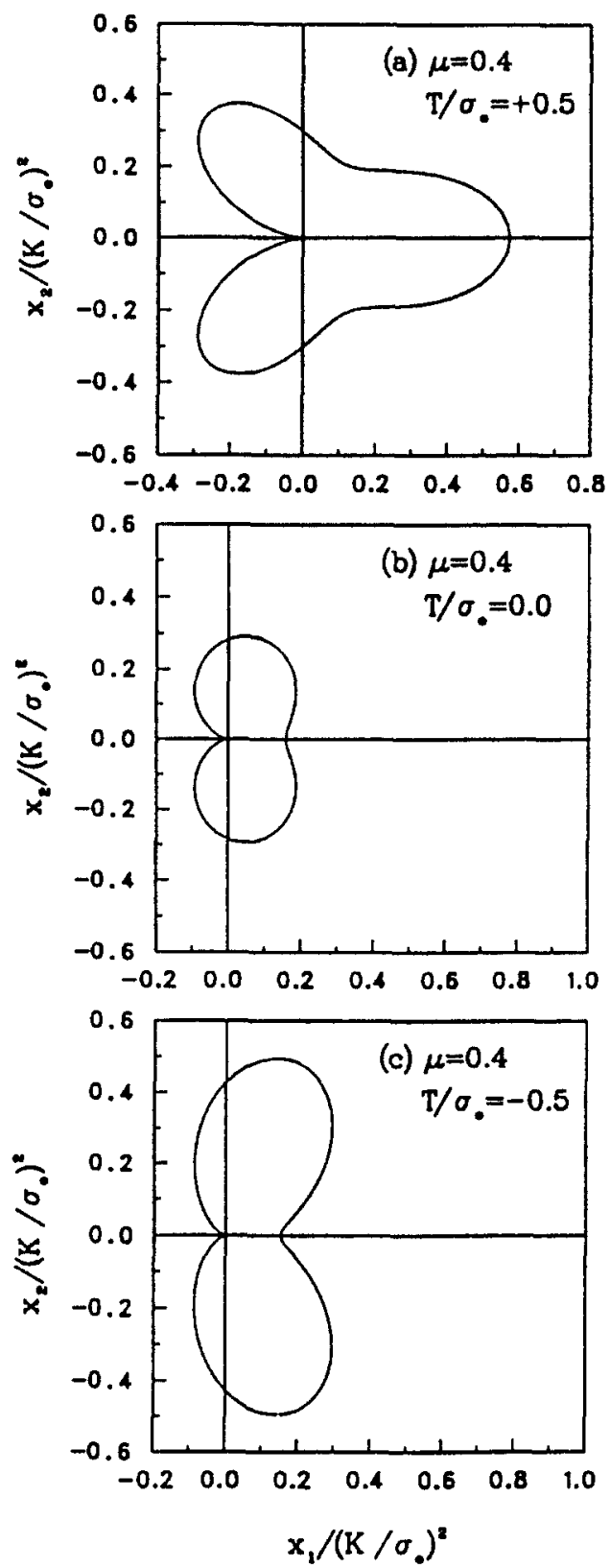

Fig. 13. The normalized plastic zone sizes and shapes obtained from the linear elastic asymptotic fields for $\mu=0.4$. (a) $T / \sigma_{0}=0.5$, (b) $T / \sigma_{0}=0$, (c) $T / \sigma_{0}=-0.5$.

for perfectly plastic materials the stresses with reference to those of the Prandtl field can be written as

$$
\sigma_{i j}=\left(\sigma_{i j}\right)_{\text {Prandtl }}+Q \sigma_{0} \delta_{i j},|\theta| \leqslant \pi / 4
$$

For pressure-sensitive materials, the fully yielded perfectly plastic solutions are given in [9]. When the sampling radius distance $2 J / \sigma_{0}$ for the stresses is well within the plastic zone at a very small ratio of $\sigma_{0} / \mathrm{E}$, the asymptotic solutions for singular plastic sectors become good 

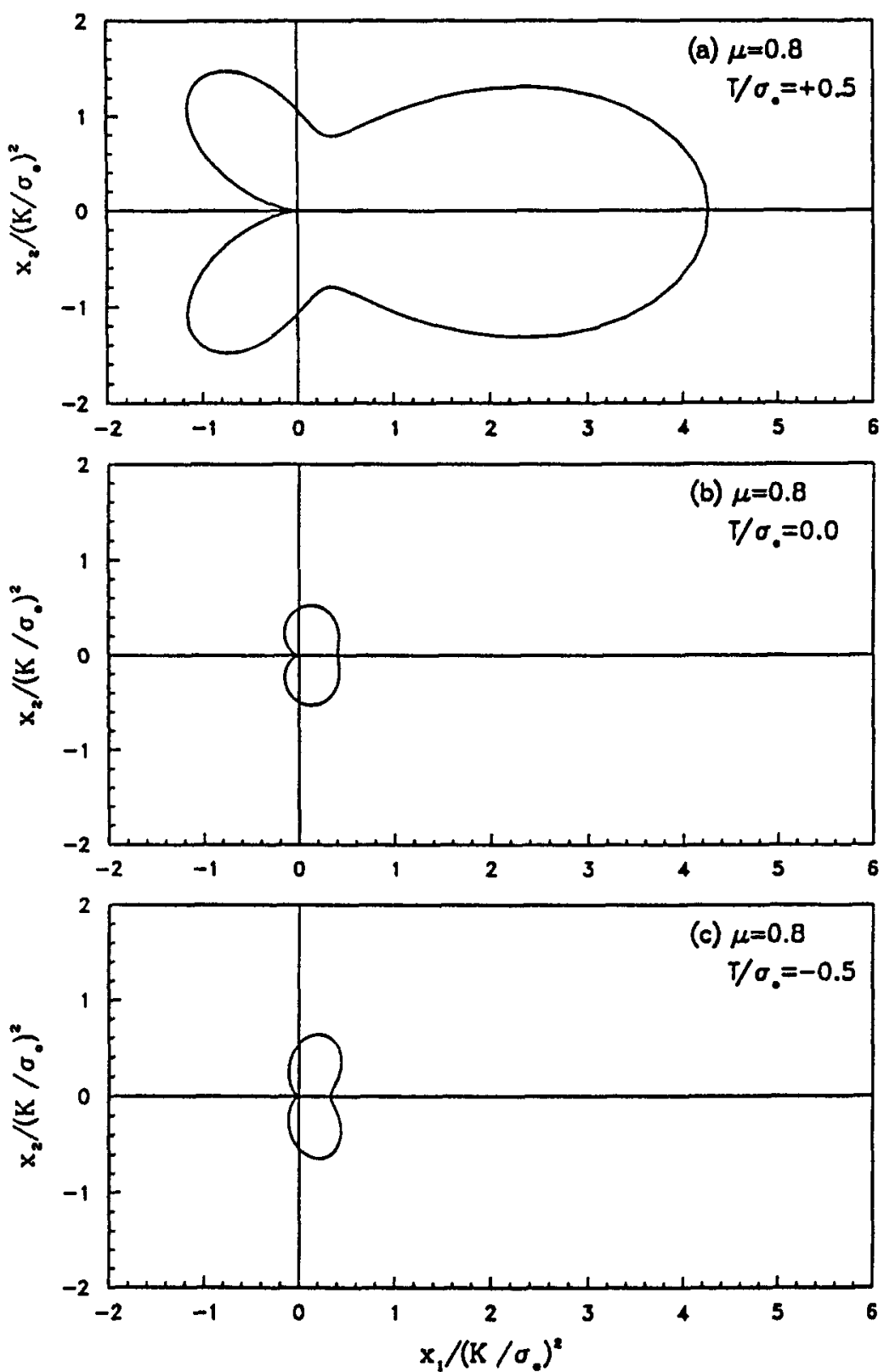

Fig. 14. The plastic zone sizes and shapes obtained from the linear elastic asymptotic fields for $\mu=0.8$. (a) $T / \sigma_{0}=0.5$, (b) $T / \sigma_{0}=0$, (c) $T / \sigma_{0}=-0.5$.

approximations. Then a generalization of the above equation for pressure-sensitive materials is

$$
\sigma_{i j}=\left(\sigma_{i j}\right)_{\mathrm{LP}}+Q_{11} \sigma_{0} \delta_{1 i} \delta_{1 j}+Q_{22} \sigma_{0} \delta_{2 i} \delta_{2 j}+Q_{33} \sigma_{0} \delta_{3 i} \delta_{3 j},|\theta| \leqslant \theta_{1},
$$

where 'LP' denote the solutions of $\mathrm{Li}$ and Pan [9], and $\theta_{1}$ is given by (48). $Q_{22}$ and $Q_{33}$ can be expressed as functions of $Q_{11}$ from (23) and (9) as

$$
Q_{22}=\frac{\left[1-\mu^{2} / 3\right]^{1 / 2}-\mu}{\left[1-\mu^{2} / 3\right]^{1 / 2}+\mu} Q_{11}
$$



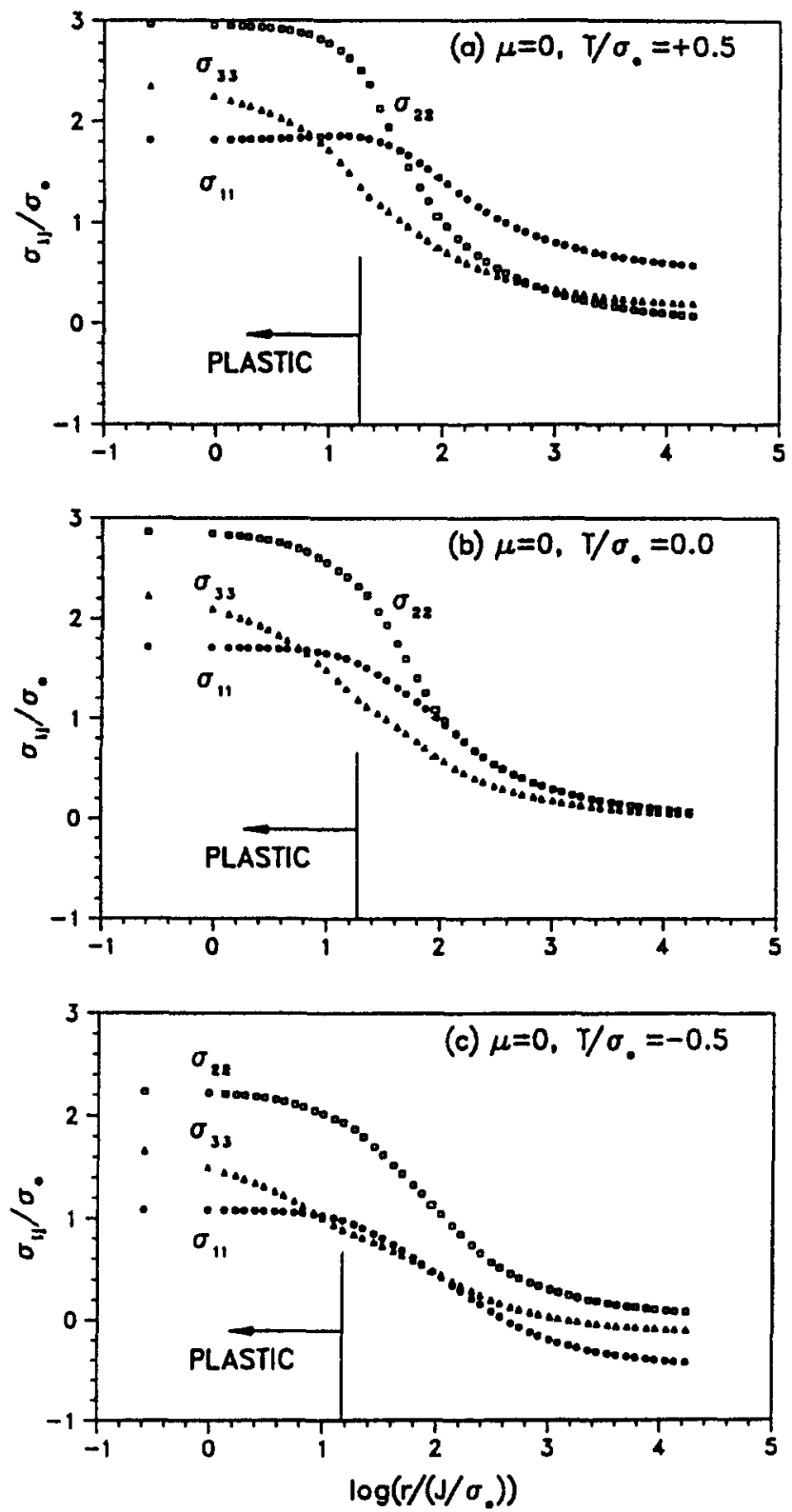

Fig. 15. The stresses normalized by $\sigma_{0}$ plotted as functions of the radial distances normalized by $J / \sigma_{0}$ in a logarithmic scale for $\mu=0$ from the finite element computations. (a) $T / \sigma_{0}=0.5$, (b) $T / \sigma_{0}=0$, (c) $T / \sigma_{0}=-0.5$.

and

$$
Q_{33}=\frac{3+2 \mu^{2}}{3\left(\left[1-\mu^{2} / 3\right]^{1 / 2}+\mu\right)\left[1-\mu^{2} / 3\right]^{1 / 2}} Q_{11} .
$$

As shown in Figs. 15, 16, and 17, the rate of change of $\sigma_{33}$ is finite for most of the cases except in the case of $\mu=0.4$ under $T / \sigma_{0}=0.5$ and 0 . Under the circumstances, we select the crack-tip fields under small-scale yielding and $T=0$ as the reference field. Then we have

$$
\sigma_{i j}=\left(\sigma_{i j}\right)_{T=0}+Q_{11} \sigma_{0} \delta_{1 i} \delta_{1 j}+Q_{22} \sigma_{0} \delta_{2 i} \delta_{2 j}+Q_{33} \sigma_{0} \delta_{3 i} \delta_{3 j},|\theta| \leqslant \theta_{1} .
$$



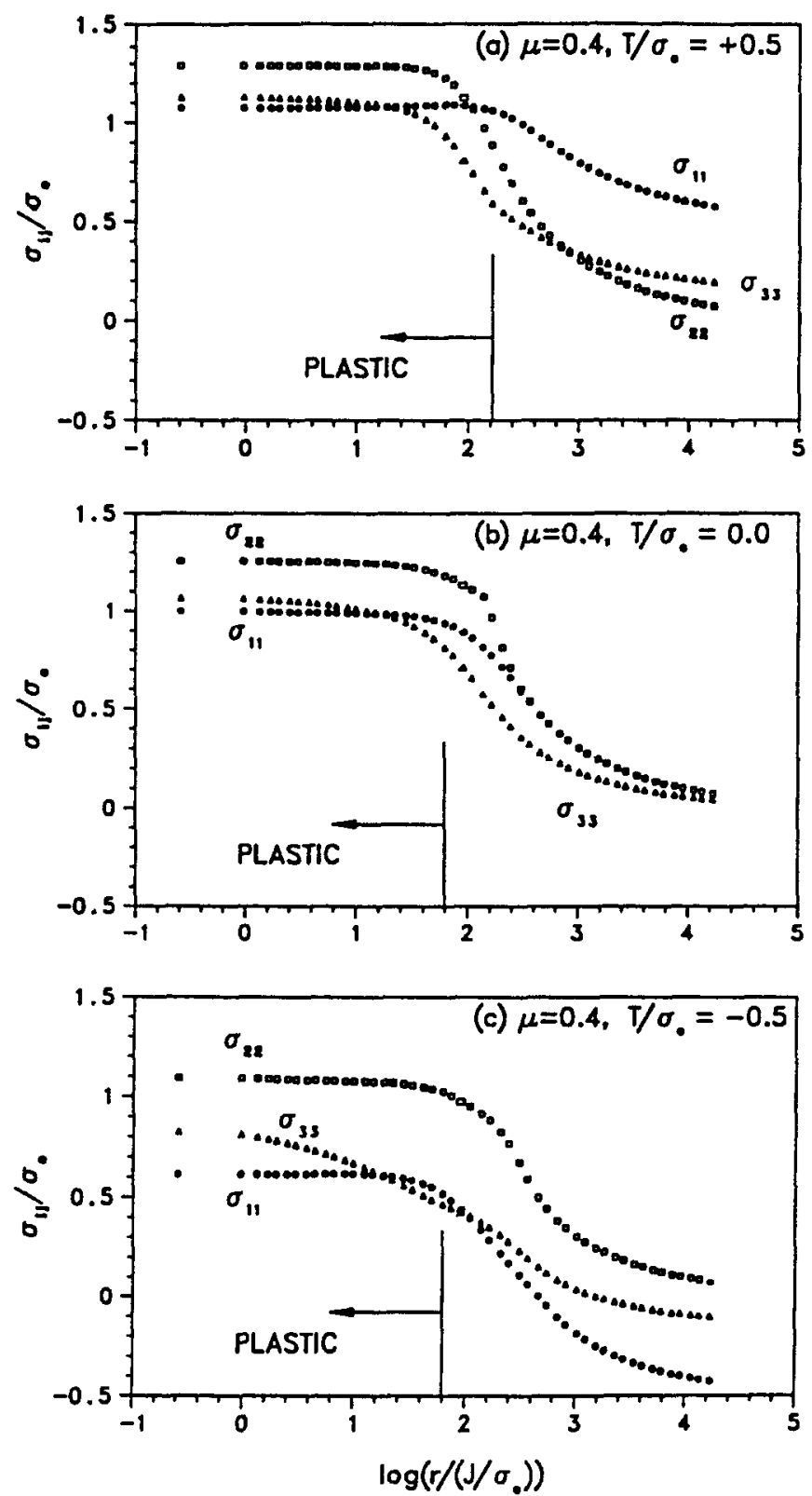

Fig. 16. The stresses normalized by $\sigma_{0}$ plotted as functions of the radial distances normalized by $J / \sigma_{0}$ in a logarithmic scale for $\mu=0.4$ from the finite element computations. (a) $T / \sigma_{0}=0.5$, (b) $T / \sigma_{0}=0$, (c) $T / \sigma_{0}=-0.5$.

The difference fields are plotted as functions of $r /\left(J / \sigma_{0}\right)$ in Fig. 18. We see positive slopes for $Q_{11}, Q_{22}$ and $Q_{33}$ for $\mu=0$. As $r$ decreases to $0.3 J / \sigma_{0}, Q_{11}, Q_{22}$ and $Q_{33}$ seem to approach to one value which agrees with the results for incompressible materials in $[21,22]$ (the value of $Q_{33}$ at $0.3 J / \sigma_{0}$ seems to be a numerical perturbation). For pressure-sensitive materials with $\mu=0.4$ and 0.8 , we do see relative flat difference fields. For $\mu=0.8$, we see some oscillation of the numerical results. For $\mu=0.4$ under $T / \sigma_{0}=0.5, Q_{11}, Q_{22}$ and $Q_{33}$ can be described by (55) and (56) quite well. This can be seen from the relative flatness of the stresses in Figs. 16(a) and (b). 

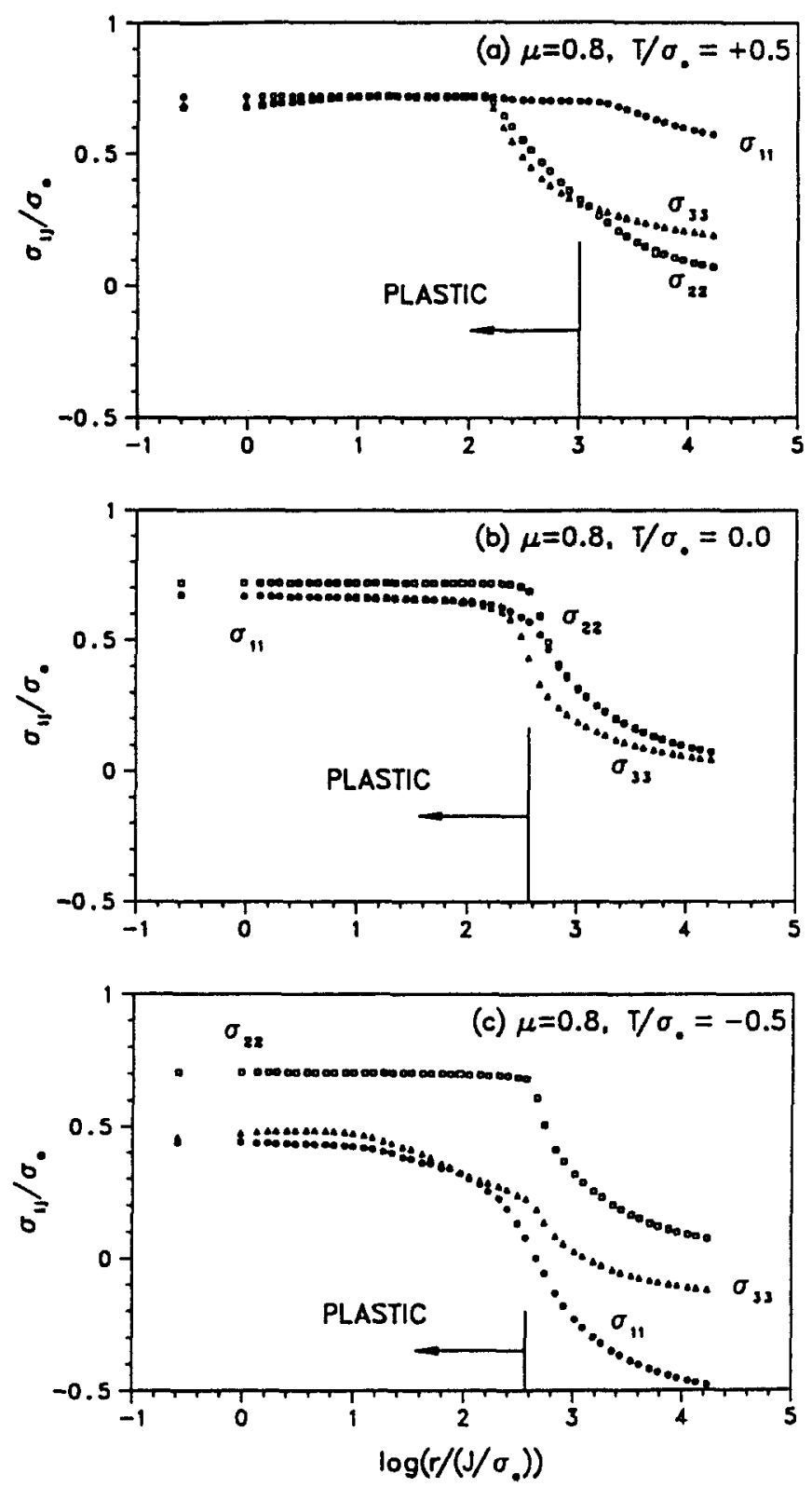

Fig. 17. The stresses normalized by $\sigma_{0}$ plotted as functions of the radial distances normalized by $J / \sigma_{0}$ in a logarithmic scale for $\mu=0.8$ from the finite element computations. (a) $T / \sigma_{0}=0.5$, (b) $T / \sigma_{0}=0$, (c) $T / \sigma_{0}=-0.5$.

In conclusion, well within the plastic zone the normalized hoop and hydrostatic stress ahead of the crack tip decrease, and the normalized plastic zone size increases as the pressure sensitivity increases. As the $T$ stress decreases, the normalized hydrostatic stress ahead of the crack tip decreases for a given $\mu$. As the $T$ stress increases, the plastic zone shifts toward the crack surfaces and expands ahead of the crack tip. For $\mu=0$ and 0.4 the plastic zones concentrate as two narrow strips inclined at certain angles to the crack line. The plastic zone sizes at these angles are much larger than those ahead of the crack tip. However, for $\mu=0.8$ under $T / \sigma_{0}=0$ and -0.5 , the plastic deformation is concentrated along the crack line. 


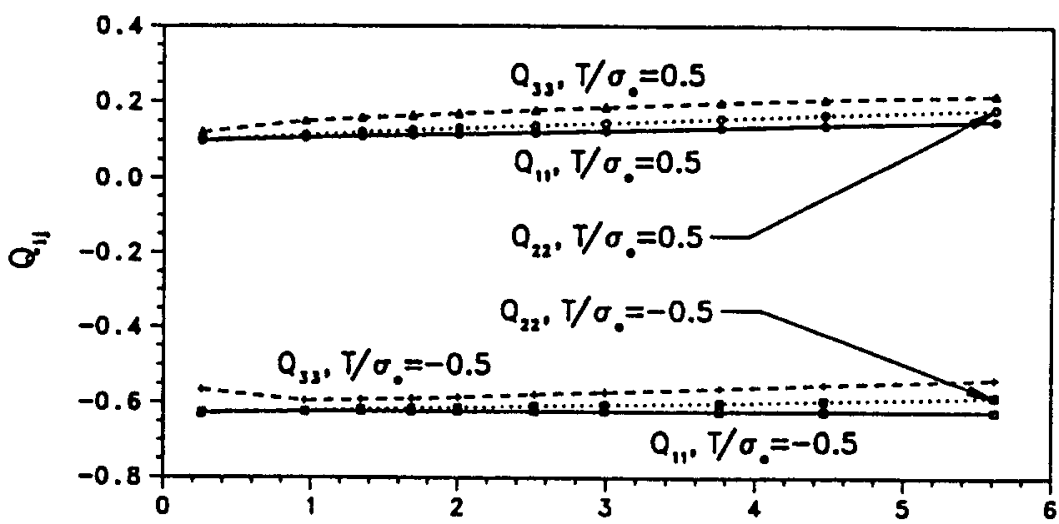

$\mu=0.0$

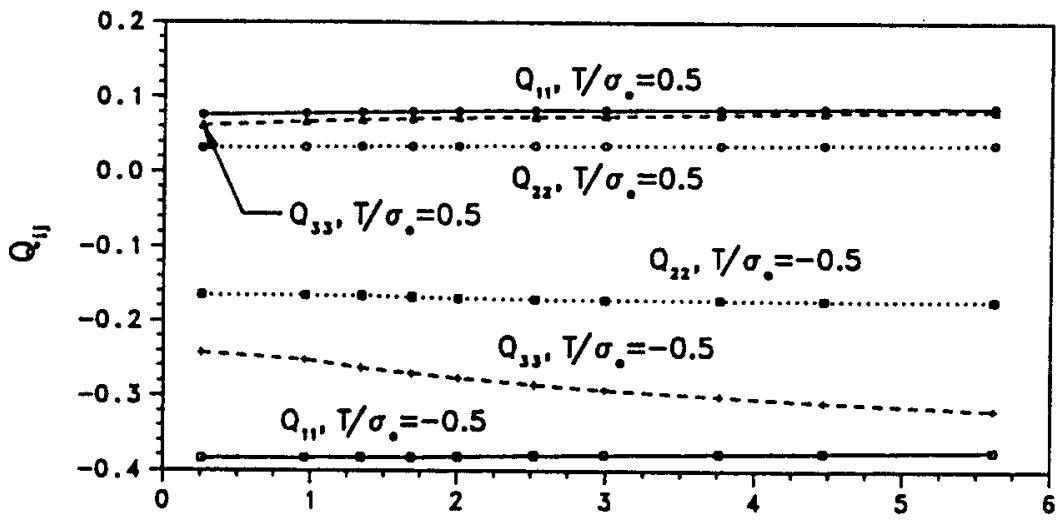

$\mu=0.4$

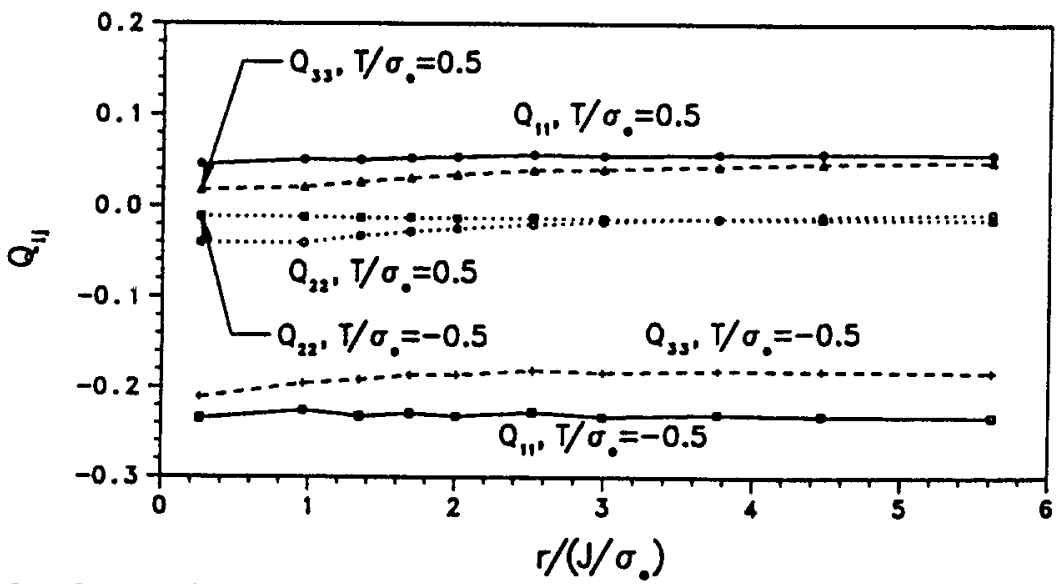

$\mu=0.8$

Fig. 18. $Q_{11}, Q_{22}$, and $Q_{33}$ plotted as functions of the radial distances normalized by $J / \sigma_{0}$ from the finite element computations for $\mu=0,0.4$ and 0.8 .

The computed stress state in the second constant stress sector which is located between the centered fan sector and the elastic sector does not agree well with the asymptotic solution in most of the cases. Specifically, the values of $\sigma_{33}$ of the finite element solutions in the second constant stress sector showed some discrepancies from those of the asymptotic solutions. If this sector is considered as a transition sector between the centered fan sector and the elastic sector, it can be regarded as a non-singular plastic sector as discussed in [23]. But the asymptotic in-plane stress distributions of this sector are sometimes very close to those of the finite element computations for several cases as shown in Figs. 3, 5 and 7. Here we allow 
the discontinuity of $\sigma_{r r}$ and $\sigma_{33}$ along the border between the second constant stress sector and the elastic sector in our asymptotic analysis. Then we can input more parameters from the computations so that the results of asymptotic analysis can be fitted well with those of the finite element computations. If we closely examine the stresses obtained from the finite element computations in and near this sector, we find that all the stresses are continuous to the neighboring sectors. Further investigation of the stresses in this transition sector is needed. At least in this paper, we show that (40)-(43) for finite stress elastic sectors can be used to fit the computational results quite well.

One of the motivations to study the near-tip fields for pressure-sensitive materials is to investigate the toughening in phase transformation materials. We can compare the phase transformation zones of the computational results with those observed in experiments. For $\mathrm{CeO}_{2}$-TZP ceramics, the phase transformation gives an initial perfectly plastic response $[6,7]$. When both the available effective and volumetric phase transformation strains are much larger than the effective strain and the volumetric strain at the inception of the phase transformation, we have the so-called strong transformation case [4, 33]. In this case, the phase transformation zone size and shape should be close to those of the computational results for elastic perfectly plastic materials presented here. Indeed, in the finite element computations with the consideration of finite phase transformation strains (or plastic strains) as occurred in phase transformation materials, the plastic zone size and shape are very close to those presented here [43]. However, these computations take much longer computational time than the computations for elastic perfectly plastic materials.

With the consideration of finite phase transformation strains, the computational results of Ben Aoun and Pan [44] showed an elongated plastic zone shape with a sharp front under plane stress conditions. The elongated phase transformation zone is remarkably close to that reported in [8] for both stationary and growing cracks. On the other hand, the phase transformation zone based on the linear elastic asymptotic crack-tip stress field and the corresponding Coulomb's phase transformation criterion has a completely different shape from that of the experimental observations as reported in [8]. Therefore this close agreement in phase transformation zone shape indicates that the constitutive law and the computational modeling presented here can be used as a tool to examine the toughening in this class of materials.

It should be noted that the height of the plastic zone has an important implication to the crack growth resistance (R-curve) behavior [5]. As shown in Figs. 9, 10, and 11 for elastic perfectly plastic materials, the height of the plastic zone can be influenced significantly by the $T$ stress. These results can be considered as approximations for strong transformation materials as discussed earlier. Another extreme case is when the phase transformation strains are extremely small when compared with the strains at the inception of the phase transformation. In this case, we have the so-called weak transformation materials $[4,33]$. As shown Figs. 12, 13, and 14 which are obtained from the linear elastic asymptotic crack-tip stress field, the height of the plastic zone can also be influenced substantially by the $T$ stress. These results can be considered as approximations for weak transformation materials. The normalized plastic zone heights from the finite element computations for elastic perfectly plastic materials and the linear elastic asymptotic crack-tip stress field are listed in Table 1 . In general as $T / \sigma_{0}$ increases or decreases from 0 , the normalized plastic zone height increases for pressure sensitive materials. For $\mu=0.8$, the plastic zone height for elastic perfectly plastic materials increases by a factor of 10 when $T / \sigma_{0}$ increases from 0 to 0.5 . However, the normalized plastic zone height from the linear elastic asymptotic crack-tip stress field only increases by a factor of 3 for $\mu=0.8$ when $T / \sigma_{0}$ increases from 0 to 0.5 . These results suggest that the 
Table 1. The maximum heights of the plastic zone normalized by $\left(K / \sigma_{0}\right)^{2}$ from the elastic-perfectly plastic finite element computations and the linear elastic asymptotic analyses

\begin{tabular}{lcll}
\hline$\mu$ & $T / \sigma_{0}$ & \multicolumn{2}{l}{ Plastic zone heights normalized by $\left(K / \sigma_{0}\right)^{2}$} \\
\cline { 3 - 4 } & & Elastic-perfectly plastic & Linear elastic \\
\hline 0 & 0.5 & 0.133 & 0.112 \\
0 & 0 & 0.133 & 0.132 \\
0 & -0.5 & 0.370 & 0.323 \\
0.4 & 0.5 & 0.446 & 0.377 \\
0.4 & 0 & 0.236 & 0.293 \\
0.4 & -0.5 & 0.383 & 0.496 \\
0.8 & 0.5 & 1.850 & 1.478 \\
0.8 & 0 & 0.191 & 0.525 \\
0.8 & -0.5 & 0.283 & 0.636 \\
\hline
\end{tabular}

$T$ stress can significantly affect the toughening due to the phase transformation, especially for strong transformation materials. Of course this is based on the assumption that the plastic zone heights in terms of $K$ and $T$ for growing cracks are similar to those for stationary cracks.

When the phase transformation strains are considered to be finite, the results in $[43,44]$ show that as the radial distance to the crack tip approaches to zero, the near-tip stress and strain fields recover the $1 / \sqrt{r}$ singularity and the amplitude of the singularity, the near-tip $K$, is the same as that of the remote applied $K$. The radial distance to the crack tip, at which the $1 / \sqrt{r}$ singularity is recovered, depends upon the magnitude of the available phase transformation strains. When the full amount of the phase transformation strains (about 4 percent volumetric strain) is considered for $\mu=0.8$, the crack-tip opening stress is about 30 times the initial generalized phase transformation stress at the radial distance where the near-tip stresses begin to be governed by the near-tip $K$ as $r$ decreases. If we assume that the material element of the transformation toughened ceramics can sustain such a high stress or more without fracture at this radial distance or smaller to the tip, then the phase transformation does not influence the near-tip stresses governed by the near-tip $K$ and consequently the initiation value of $K$. However, the phase transformation does affect the R-curve behavior of growing cracks due to the plastic wake. If the critical stress and/or strain at fracture are determined at the critical length scale larger than the radial distance where the near-tip $K$ is dominant, then the phase transformation strains can affect the initiation value of $K$. This should be investigated further for different transformation toughened ceramics.

The values of $K$ at fracture initiation and the R-curve behaviors are quite different under plane strain conditions and under plane stress conditions. Under plane strain conditions, the $T$ stress has significant effects on the hydrostatic stress ahead of the tip due to the asymptotic structure of the crack-tip fields for perfectly plastic materials. On the other hand, under plane stress conditions the $T$ stress does not affect the stresses ahead of the crack tip for perfectly plastic materials because of a centered fan sector located ahead of the tip $[47,44]$. But the $T$ stress does affect the plastic zone size and shapes for perfectly plastic materials under plane stress conditions. When the finite phase transformation strains are considered, the general trend of the radial dependence of the crack-tip stresses is the same as that under plane 
strain conditions. When the full amount of the phase transformation strains (about 4 percent volumetric strain) is considered for $\mu=0.8$, the crack-tip opening stress is about 100 times the initial generalized phase transformation stress at the radial distance where the near-tip stresses begin to be governed by the near-tip $K$ as $r$ decreases [44]. The above discussion on the effect of phase transformation on the initiation value of $K$ and the $\mathrm{R}$-curve behavior under plane strain conditions should apply to the plane stress conditions but with the understanding that the crack-tip hydrostatic stress is lower under plane stress conditions.

\section{Appendix}

In this appendix, we will show that all the stress components under plane strain conditions must be continuous along the border between a singular constant stress sector and a singular centered fan sector. First, we combine the plane strain condition in (9) and the yield condition in (10) for singular plastic sectors and we arrive at a quadratic equation for $\sigma_{r r}$ in terms of $\sigma_{r \theta}$ and $\sigma_{\theta \theta}$ as

$$
A \sigma_{r r}^{2}+B \sigma_{r r}+C=0
$$

where

$$
\begin{aligned}
& A=1 / 4-\mu^{2} / 3 \\
& B=\left(-1 / 2-\mu^{2} / 3\right) \sigma_{\theta \theta}+\mu \sigma_{0} / \sqrt{3} \\
& C=\left(1 / 4-\mu^{2} / 3\right) \sigma_{\theta \theta}^{2}+\left(\mu \sigma_{0} / \sqrt{3}\right) \sigma_{\theta \theta}+\left(1-\mu^{2} / 3\right) \sigma_{r \theta}^{2}-\sigma_{0}^{2} / 3
\end{aligned}
$$

If $1 / 4-\mu^{2} / 3 \neq 0$ and

$$
B^{2}-4 A C=\left(1-\frac{\mu^{2}}{3}\right)\left(\left(\mu \sigma_{\theta \theta}-\frac{\sigma_{0}}{\sqrt{3}}\right)^{2}-\left(1-\frac{4}{3} \mu^{2}\right) \sigma_{r \theta}^{2}\right) \geqslant 0
$$

Eqn. (A.1) gives real roots for $\sigma_{r r}$ in terms of $\sigma_{\theta \theta}$ and $\sigma_{r \theta}$. In general, when $B^{2}-4 A C>$ 0 , we should have two real roots for $\sigma_{r r}$. This is the reason that we can have a radial stress discontinuity between two constant stress sectors (see some results on radial stress discontinuity for the Mises materials in $[34,35])$. When $B^{2}-4 A C=0$ and $\mu \neq \sqrt{3}$, then

$$
\left(\mu \sigma_{\theta \theta}-\frac{\sigma_{0}}{\sqrt{3}}\right)^{2}-\left(1-\frac{4}{3} \mu^{2}\right) \sigma_{r \theta}^{2}=0
$$

Under this condition, we have only one double root for (A.1)

$$
\sigma_{r r}=\frac{2}{1-\frac{4}{3} \mu^{2}}\left[\left(\frac{1}{2}+\frac{\mu^{2}}{3}\right) \sigma_{\theta \theta}-\frac{\mu \sigma_{0}}{\sqrt{3}}\right] .
$$

We have closed form solutions in (32) through (34) for centered fan sectors. From the solutions of $\sigma_{r \theta}$ and $\sigma_{\theta \theta}$ in (32) and (33), the condition in (A.3) can easily be shown to be satisfied. Therefore in centered fan sectors where an extra condition $P_{r r}=0$ must be enforced, $\sigma_{r r}$ 
must be uniquely determined from $\sigma_{r \theta}$ and $\sigma_{\theta \theta}$ as a double root. This actually leads to a linear relation between $\sigma_{r r}$ and $\sigma_{\theta \theta}$ as in (A.4).

Along the border between a constant stress sector and a centered fan sector, $\sigma_{r \theta}$ and $\sigma_{\theta \theta}$ must be continuous due to the equilibrium. Then $\sigma_{r \theta}$ and $\sigma_{\theta \theta}$ of the constant stress sector must satisfy (A.3). On both sides of the border, $\sigma_{r r}$ is linearly related to $\sigma_{\theta \theta}$ as in (A.4.) From (9) $\sigma_{33}$ now can be uniquely determined as a function of $\sigma_{r \theta}$ and $\sigma_{\theta \theta}$. Therefore we can say that along the border of a singular plastic constant stress sector and a singular plastic centered fan sector, when $\sigma_{\theta \theta}$ and $\sigma_{r \theta}$ are continuous due to the equilibrium equations under the plane strain conditions, $\sigma_{r r}$ and $\sigma_{33}$ must be continuous. This full stress continuity condition between a constant stress sector and a centered fan sector should be valid for any quadratic yield condition by following the above general argument, for example, see the crack-tip fields based on Hill's quadratic yield conditions $[45,46]$.

\section{Acknowledgement}

The financial support of the work was provided by the National Science Foundation under grant number DMR-8708405. Helpful discussions with Dr. P. Dong of Edison Welding Institute of Columbus, Ohio are also appreciated.

\section{References}

1. D. C. Drucker, Metallurgical Transactions 4 (1973) 667-673.

2. W. A. Spitzig and O. Richmond, Polymer Engineering and Science 19 (1979) 1129-1139.

3. L. M. Carapellucci and A. F. Yee, Polymer Engineering and Science 26 (1986) 920-930.

4. B. Budiansky, J. W. Hutchinson and J. C. Lambropoulos, International Journal of Solids and Structures 19 (1981) 337-355.

5. R. M. McMeeking and A. G. Evans, Journal of the American Ceramic Society 65 (1982) 242-246.

6. I.-W. Chen and P. E. Reyes Morel, Journal of the American Ceramic Society 69 (1986) 181-89.

7. P. E. Reyes Morel and I.-W. Chen, Journal of the American Ceramic Society 71 (1988) 343-353.

8. C.-S. Yu and D. K. Shetty, Journal of the American Ceramic Society 72 (1989) 921-928.

9. F. Z. Li and J. Pan, Journal of Applied Mechanics 57 (1990) 40-49.

10. F. Z. Li and J. Pan, Engineering Fracture Mechanics 35 (1990) 1105-1116.

11. J. W. Hutchinson, Journal of the Mechanics and Physics of Solids 16 (1968) 13-31.

12. Ibid, 337-347.

13. J. R. Rice and G. F. Rosengren, Journal of the Mechanics and Physics of Solids 16 (1968) 1-12.

14. J. Pan and I. W. Chen, Crack-Tip Fields for Transformation Toughening Materials, presented at the 93rd Annual Meeting of the American Ceramic Society, Cincinnati, Ohio (1991).

15. P. Dong and J. Pan, International Journal of Solids and Structures 28 (1991) 1113-1127.

16. S. G. Larsson and A. J. Carlsson, Journal of the Mechanics and Physics of Solids 21(1973) 263-277.

17. J. R. Rice, Journal of the Mechanics and Physics of Solids 22 (1974) 17-26.

18. C. Bentegón and J. W. Hancock, Journal of Applied Mechanics 58 (1991) 104-1 10.

19. A. M. Al-Ani and J. W. Hancock, Journal of the Mechanics and Physics of Solids 39 (1991) 23-43.

20. Z.-Z. Du and J. W. Hancock, Journal of the Mechanics and Physics of Solids 39 (1991) 555-567.

21. N. P. O'Dowd and C. F. Shih, Journal of the Mechanics and Physics of Solids 39 (1991) 989-1015.

22. Ibid, 40 (1992) 939-963.

23. J. R. Rice, in Mechanics of Solids: The R. Hill 60th Anniversary Volume, H. G. Hopkins and M. J. Sewell (eds.), Pergamon Press, Oxford (1982) 539-562.

24. D. C. Drucker and W. Prager, Quarterly of Applied Mathematics 10 (1952) 157-165.

25. J. W. Rudnicki and J. R. Rice, Journal of the Mechanics and Physics of Solids 23 (1975) 371-394.

26. A. Needleman and J. R. Rice, in Mechanics of Sheet Metal Forming D. P. Koistinen and N.-M. Wang (eds.), Plenum Publishing Co. (1978) 237-265.

27. H.-Y. Jeong, X.-W. Li, A. F. Yee and J. Pan, Mechanics of Materials, in press (1994).

28. H.-Y. Jeong, A Macroscopic Constitutive Law for Porous Solids with Pressure-Sensitive Matrices and Its Implications for Plastic Flow Localization and Crack-Tip Behavior, Ph.D. thesis, The University of Michigan, Ann Arbor, Michigan (1992). 
29. R. Hill, The Mathematical Theory of Plasticity, Clarendon Press, Oxford (1950).

30. T. H. Wu, Soil Mechanics. Allyn and Bacon, Boston, Massachusetts (1966).

31. O. Richmond and W. A. Spitzig, International Union of Theoretical and Applied Mechanics (1980) 377-286.

32. A. J. Kinloch and R. J. Young, Fracture Behaviour of Polymers, Elsevier Applied Science, London and New York (1983).

33. I. W. Chen, Journal of the American Ceramic Society 74 (1991) 2564-2572.

34. P. Dong and J. Pan, Engineering Fracture Mechanics 37 (1990) 43-57.

35. P. Dong and J. Pan, International Journal of Fracture 45 (1990) 243-262.

36. J. R. Rice, Journal of Applied Mechanics 35 (1968) 379-386.

37. J. R. Rice and D. M. Tracey, in Numerical and Computer Methods in Structural Mechanics, S. J. Fenves et al. (eds), Academic Press (1973) 585-623.

38. P. S. Leevers and J. C. Radon, International Journal of Fracture 19 (1982) 311-325.

39. R. M. McMeeking, Journal of the Mechanics and Physics of Solids 25 (1977) 357-381.

40. C. F. Shih, Journal of the Mechanics and Physics of Solids 29 (1981) 305-326.

41. F. A. McClintock, in Fracture: An Advanced Treatise, Vol. 3, Engineering Fundamentals and Environmental Effects, H. Liebowitz (ed.) Academic Press, New York (1971) 47.

42. D. M. Tracey, Journal of Engineering Materials and Technology 98 (1976) 146-151.

43. M. Kim and J. Pan, Effects of Non-Singular Stresses on Crack-Tip Fields for Transformation Toughened Materials: Part I-Plane Strain Case, presented at the 95th Annual Meeting of the American Ceramic Society, Cincinnati, Ohio (1993).

44. Z. E. A. Ben-Aoun and J. Pan, Effects of Non-Singular Stresses on Crack-Tip Fields for Transformation Toughened Materials: Part II-Plane Stress Case, presented at the 95th Annual Meeting of the American Ceramic Society, Cincinnati, Ohio (1993).

45. J. Pan, Journal of the Mechanics and Physics of Solids 34 (1986) 263-277.

46. R. Hill, Proceedings of the Royal Society of London A341 (1948) 281-297.

47. Z. E. A. Ben-Aoun and J. Pan, Engineering Fracture Mechanics 44 (1993) 649-662. 